\title{
A optical fiber double scrambler and mechanical agitator system for the Keck planet finder spectrograph
}

Martin M. Sirk, Edward H. Wishnow, Marie Weisfeiler, Elisha Jhoti, James Curtis, et al.

Martin M. Sirk, Edward H. Wishnow, Marie Weisfeiler, Elisha Jhoti, James Curtis, Yuzo Ishikawa, Daniel Finstad, Timothy O'Hanlon, Steven R. Gibson, Jerry Edelstein, Samuel Halverson, Arpita Roy, Andrew Howard, "A optical fiber double scrambler and mechanical agitator system for the Keck planet finder spectrograph," Proc. SPIE 10702, Ground-based and Airborne Instrumentation for Astronomy VII, 107026F (6 July 2018); doi: $10.1117 / 12.2312945$

Event: SPIE Astronomical Telescopes + Instrumentation, 2018, Austin, Texas, United States 


\title{
An Optical Fiber Double Scrambler and Mechanical Agitator System for the Keck Planet Finder Spectrograph
}

\author{
Martin M. Sirk ${ }^{\mathrm{a}}$, Edward H. Wishnow ${ }^{\mathrm{a}}$, Marie Weisfeiler ${ }^{\mathrm{a}}$, Elisha Jhoti ${ }^{\mathrm{b}}$, James Curtis ${ }^{\mathrm{c}}$, Yuzo \\ Ishikawa $^{\mathrm{a}, \mathrm{d}}$, Daniel Finstad ${ }^{\mathrm{e}}$, Timothy O'Hanlon ${ }^{\mathrm{a}}$, Steven R. Gibson ${ }^{\mathrm{a}}$, Jerry Edelstein ${ }^{\mathrm{a}}$, Samuel \\ Halverson ${ }^{\text {f,g,*}}$, Arpita Roy $^{\mathrm{h}}$, and Andrew Howard ${ }^{\mathrm{h}}$

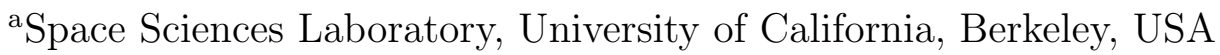 \\ ${ }^{\mathrm{b}}$ University of Edingburgh, Edinburgh, UK \\ ${ }^{\mathrm{c}}$ Lawrence Berkeley National Laboratory, University of California, Berkeley, USA \\ dSan Francisco State University, San Francisco, USA \\ ${ }^{e}$ Dept of physics, Syracuse University, Syracuse, USA \\ ${ }^{\mathrm{f}}$ University of Pennsylvania, Philidelphia, USA \\ gMassachusetts Institute of Technology, Cambridge, USA \\ ${ }^{\mathrm{h}}$ California Institute of Technology, Pasadena, USA \\ *NASA Sagan Fellow
}

\begin{abstract}
We present the design and test results of a double-scrambler and fiber agitator system for the Keck Planet Finder (KPF) spectrograph. The mechanical agitator for modal noise suppression is constructed from two linear stages with the fibers mounted in a "W" curve. When driven back-and-forth at different rates, the stages change the position of the fiber curves, and hence vary the modes propagating through the fiber. Near-field temporal centroid shifts caused by modal-noise are reduced by a factor of 100 by the agitator, while mid-range spatial frequencies have reduced power by a factor of $\sim 300$ in the near-field, and 1000 in the far-field. The scrambling system incorporates two octagonal fibers, and a scrambler consisting of two identical cemented lenses $\sim 20 \mathrm{~cm}$ apart, which exchanges the optical near- and far-fields of the fibers. The scrambler shows scrambling gains $>16,000$ in the near-field, and $>40,000$ in the far-field. The measured throughput efficiency of $99.3 \%$ of the expected maximum demonstrates that scrambler-induced focal ratio degradation (FRD) is minimal. The scrambler also serves as the feed-through into the vacuum chamber where the spectrograph is housed, thereby removing concerns about stressing the fibers, and introducing FRD, at this interface. Our illumination stabilization system, consisting of two octagonal fibers, a two lens scrambler, and a mechanical agitator, produces very homogeneous fiber output in both the near and far-fields. When coupled to the Keck Planet Finder spectrograph, this system provides illumination stability corresponding to a velocity of $0.30 \mathrm{~m} \mathrm{~s}^{-1}$.
\end{abstract}

Keywords: astronomical instruments: spectrographs, techniques: radial velocity, techniques: spectroscopic, fibers: modal-noise, fibers: scrambling

\section{INTRODUCTION}

Astronomers are on the verge of discovering Earth-sized planets via Doppler radial velocity measurements, but reliable detection and mass estimates will require instrument precision greater than is currently available. The expected radial velocity of an Earth-like planet orbiting a Sun-like star in the habitable-zone is a few $\mathrm{cm} \mathrm{s}^{-1}$, fully an order of magnitude smaller than that typically achievable by Doppler spectrographs. Non-uniform illumination of the spectrograph entrance slit, which alters the shape of the instrument point spread function (PSF), is a major precision-limiting factor in Doppler spectroscopy. ${ }^{1}$ The primary causes of non-uniform illumination at the slit are guiding errors, seeing conditions, pupil instabilities, atmospheric dispersion, and optical aberrations.

Further author information: (Send correspondence to M.M.S.)

M.M.S.: E-mail: sirk@ssl.berkeley.edu

Ground-based and Airborne Instrumentation for Astronomy VII, edited by Christopher J. Evans, Luc Simard, Hideki Takami, Proc. of SPIE Vol. 10702, 107026F · @ 2018 SPIE · CCC code: 0277-786X/18/\$18 · doi: 10.1117/12.2312945 
The use of optical fibers to inject light into spectrographs has long been known to improve spatial illumination uniformity. ${ }^{2}$ However, because light propagates through a fiber in a finite set of modes, it undergoes interference when exiting the fiber. The pattern of interference, referred to as speckle or modal-noise, changes with every motion of the fiber resulting in time-varying illumination instability. Thus, the use of a single fiber is inadequate for high precision spectroscopy.

The reduction of spatially varying illumination at the output of a fiber relative to that at the input is called 'scrambling', and a device designed to achieve this a 'scrambler'. While a single fiber provides some degree of scrambling, a 'double scrambler' consisting of an input fiber, one or two lenses, and an output fiber yields far greater scrambling. Optical scramblers, however, have no effect on modal-noise. Since changes in fiber position and shape cause changes in the speckle pattern, it is natural to mechanically move the fiber in some manner such that the fiber cycles through as many different modes as possible within a given exposure time. Such devices designed to achieve this are called 'agitators' and they are very effective at averaging out time-varying modal-noise. ${ }^{3}$

The Keck Planet Finder (KPF) spectrograph integrates several strategies to achieve high-precision radial velocities (see Gibson et al. 2018, this volume). ${ }^{4}$ The optical bench and all reflective optics are made of low thermal expansion Zerodur. The entire spectrograph will be housed in a vacuum chamber to minimize the effects caused by changes in atmospheric temperature and pressure. Illumination homogeneity of the spectrograph is ensured by a double-fiber, double-lens scrambler coupled to a two-arm agitator - the design and performance of these components is the subject of this paper. Tests are conducted at University of California Berkeley's Space Sciences Laboratory (SSL). A schematic of our fiber test apparatus is shown in Figure 1. We distinguish between the "near-field" illumination pattern which is the pattern of light at the output fiber tip, and the "far-field" pattern which is the image at a pupil downstream of the output fiber tip.

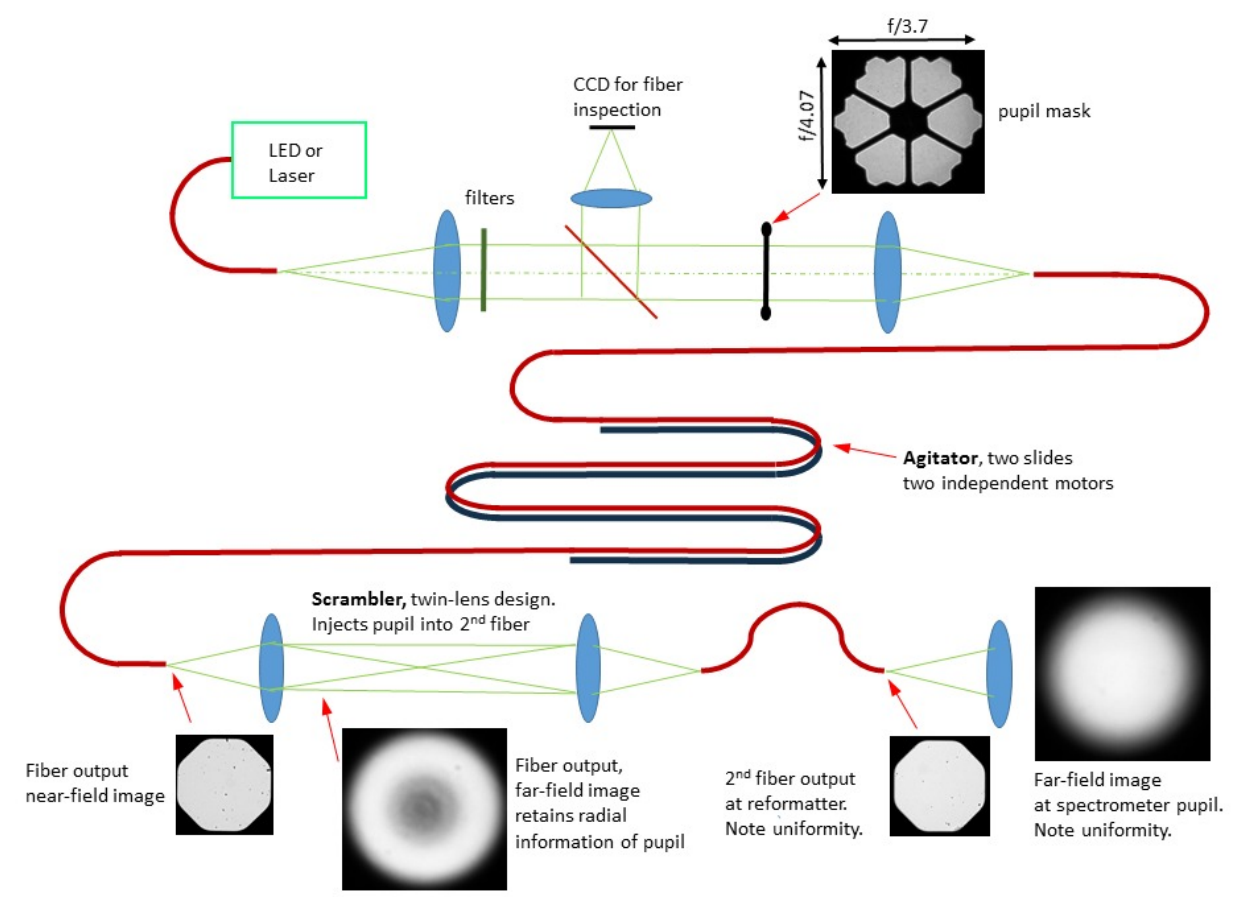

Figure 1. Test setup overview. Laser or LED light is fiber-fed to a collimator (upper left). Parallel light then passes through a pupil mask and lens which mimic the Keck secondary mirror obstruction and f/\#, and is then focused onto the input fiber tip. The fiber passes through the triple curve of the agitator and terminates at the input end of the scrambler. Light passes through the scrambler onto the output fiber and the output light inspected via a microscope and CCD camera (near-field), or by directly shining onto the CCD (far-field). 


\section{AGITATOR}

\subsection{Importance of Modal Noise Mitigation}

Light travels via modes in an optical fiber with the potential of interference between modes at the fiber exit. As described in Mahadevan et al. (2014), ${ }^{5}$ the number of excited modes depends directly on the squares of the diameter $(d)$ and numerical aperture (NA) of the fiber, and inversely on the square of the wavelength $(\lambda)$ :

$$
M=0.5\left[\frac{\pi d(\mathrm{NA})}{\lambda}\right]^{2}
$$

The modes are manifest as a speckle pattern across the near-field and far-field of a fiber. This pattern is nonuniform both spatially and temporally, and small changes in the position of the fiber, wavelength, or injection conditions can cause the speckle pattern to change. ${ }^{6}$ This non-uniformity has implications for precision radial velocity measurements, in that the speckle pattern affects the illumination of the spectrometer and can shift the centroid of the resolution element, leading to systematic Doppler errors that cannot be calibrated out with a wavelength reference.

We note the distinction presented in Halverson et al. $(2015)^{7}$ between scrambling and mitigation of fiber modal noise. Scrambling disrupts the correlation between star movement at the input of a fiber and the illumination pattern in the fiber output. It is mitigated in a static sense, via optical fibers and lens-based double scrambler systems. Conversely, modal noise requires dynamic correction, either via fiber agitation or by dynamically varying the input conditions such as using a moving diffuser, ${ }^{5}$ or a rotating mirror. ${ }^{8}$ Varying the input conditions tends to be low in throughput efficiency, and hence is more suited for calibration systems, where typically more light is available than from the science target.

Given the sensitivity of modal patterns to fiber movement, one strategy to mitigate modal noise is to agitate the fiber during an exposure. With this approach the detector records not a single speckle pattern, but rather an average of rapidly changing speckle patterns. Examples of modal noise in near-IR stellar spectra with and without fiber agitation are shown in Plavchan et al. (2013). ${ }^{9}$ Other analysis of modal noise spatial frequency for circular fibers was performed by McCoy et al. (2012), ${ }^{10}$ and for octagonal fibers by Roy et al. (2014). ${ }^{3}$

\subsection{Agitator Design}

The primary requirements for the agitator system are that it be remotely turned on and off, that its correct operation be verified via telemetry, and that it not harm the fiber by bending at too small a radius, or by binding or abrasion. During our preliminary design phase, we have designed and built a fiber agitator (shown in Figure 2) based on the system described in Roy et al. (2014). ${ }^{3}$ This mechanism employs two linear stages to change the shape of the fiber path, which follows a stylized "W". Note that during an agitator cycle the fiber path changes shape, but does not change in length.

The KPF agitator utilizes two off-the-shelf Velmex linear stages driven by stepper motors controlled by Velmex controllers. Attached to the carts on the linear stages via custom brackets is an IGUS chain (the same chain used to house movable cables in industrial applications) to hold the optical fibers. For laboratory testing, the system was mounted on a frame made of bolted-together aluminum extrusions.

We chose to place the agitator horizontal so that fibers are fully supported along their path through the agitator (as opposed to a vertical arrangement where the fibers would dangle as they pass through a bend). We originally considered agitating the entire fiber cable (with multiple fibers within), but decided a safer approach was to break the fibers out of the main cable shroud and run them through the agitator within smaller diameter flexible tubing. To further protect the fibers each fiber will be supported by its own separate platform within the IGUS chain. There are three separate sections (Figure 3) where the fibers may run such that they pass through the chain without any risk of binding or grabbing between fibers. The fiber housing is fixed at the fiber entrance and exit points (see Figure 2), but within the IGUS chain they will simply rest on the interior platforms. The bend radii of two of the turns within the IGUS chain sections is $11 \mathrm{~cm}$ while that of the third is adjustable from 11 to $15 \mathrm{~cm}$.

Each motor is independently controlled so that each linear stage can run at a differing rate and amplitude to ensure all modes are averaged out from the fiber agitation. For lab testing, each of the Velmex motors 


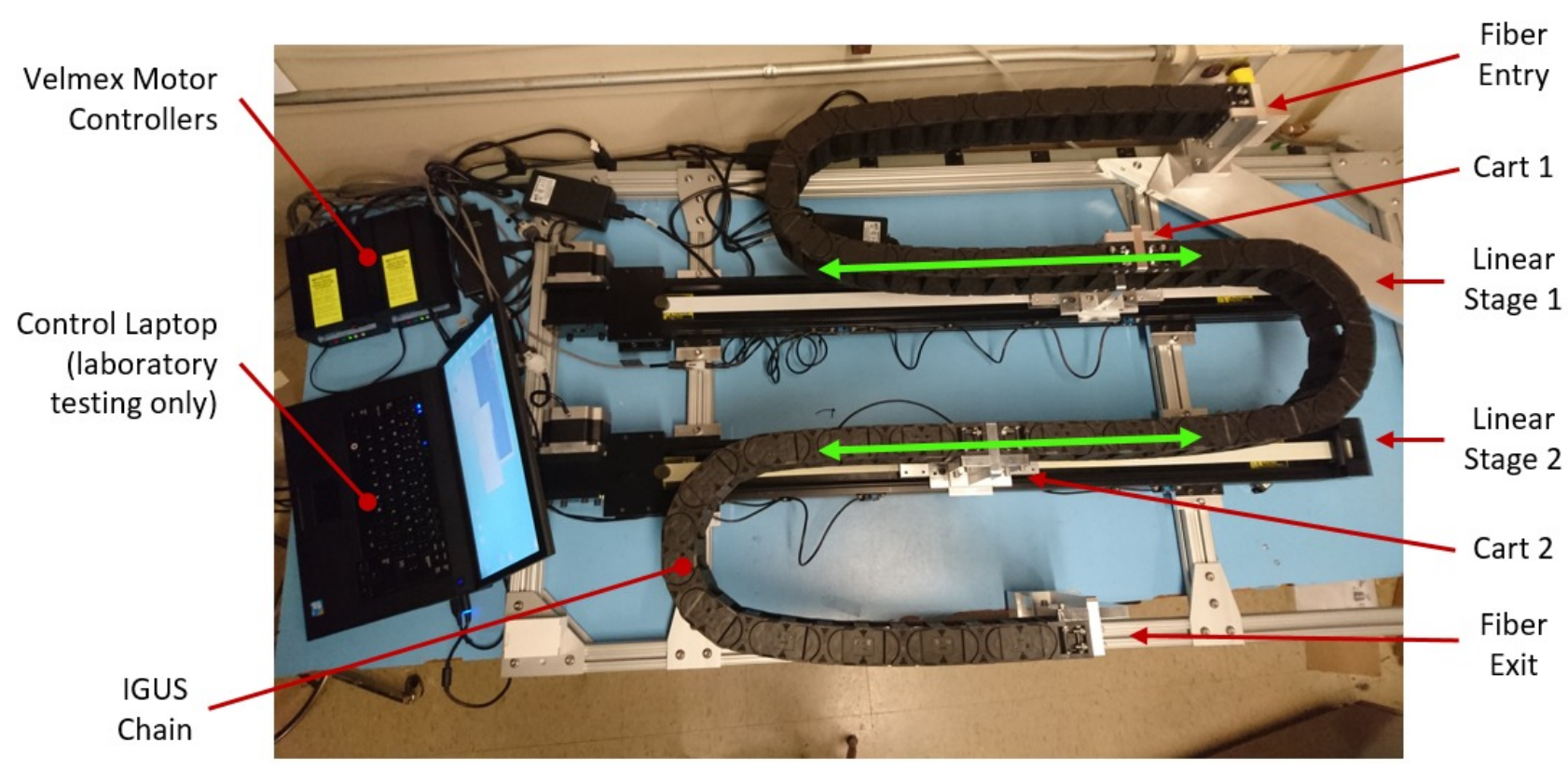

Figure 2. The KPF fiber agitator (for modal noise suppression).

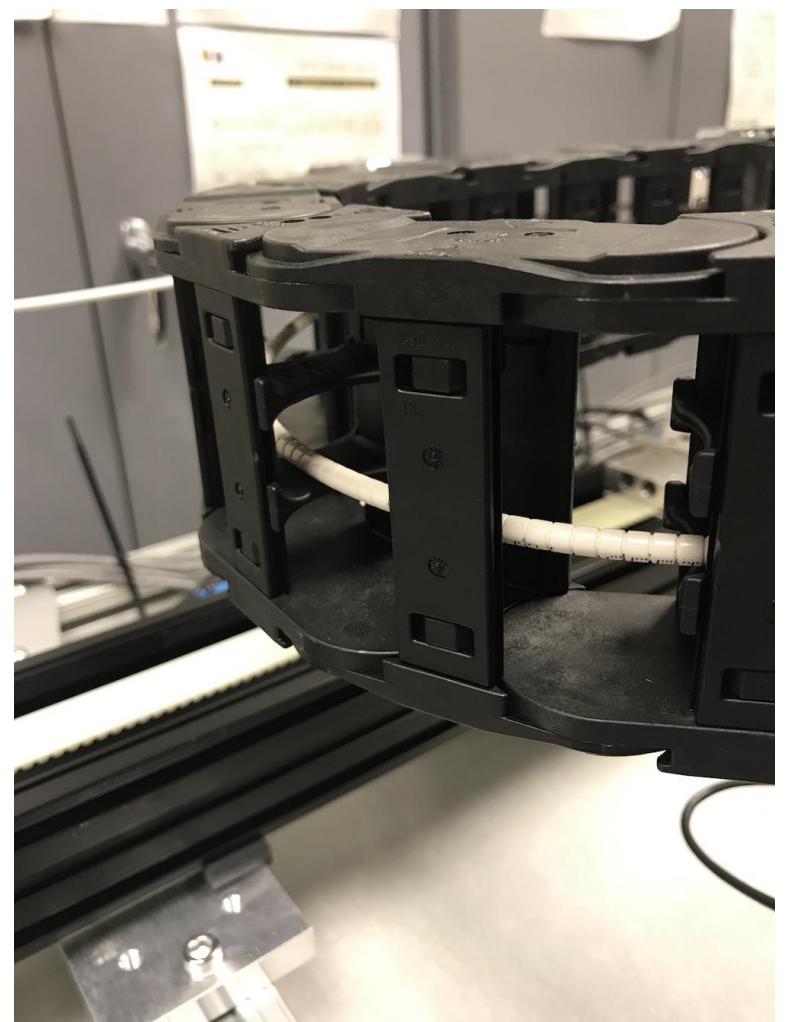

Figure 3. A single fiber within its protective tubing sitting on the middle platform within the agitator IGUS chain.

was controlled by a Python GUI that allowed adjustment of the cycle amplitude and frequency of the agitator motion. During use at the observatory, the final motion and motor parameters will be stored in the Velmex controllers, with the instrument control software simply turning the stages on or off. The mode-mixing laboratory measurements described below were made with $300 \mathrm{~mm}$ peak-to-peak motions of the two stages. They are driven 
with slightly different motor speeds, 400 and 410 steps/sec, respectively.

We considered the lifetime of our optical fiber which is subject to a number of dynamic and static bends. The fiber will have static bends for routing between the fiber injection unit (FIU) and the spectrograph and dynamic bends at the azimuth rotation cable wrap and in the fiber agitator unit. Less than a few meters of fiber will be subject to a minimum $10 \mathrm{~cm}$ bending radius. The mechanical reliability of fiber is described by statistical fatigue where flaws subjected to sufficient tensile stress grow to a failure point. Fatigue increases with initial crack size, stress, and humidity and the failure probability increases with the fiber length under stress. According to failure studies,${ }^{11}$ one can safely stress optical fiber to $1 / 5$ th of its proof stress for a continuous 25 year in-service with no expected breaks for any fiber length. Our $290 \mu \mathrm{m}$ diameter clad fiber when bent at a $100 \mathrm{~mm}$ radius will have a stress of $12 \mathrm{kpsi}$ and shall be proof tested to $100 \mathrm{kpsi}$. It therefore has a life exceeding 25 years with predicted failure probability of $<1 \times 10^{-7}$. Regardless, we shall provide a fully redundant fiber cable assembly for installation at Keck to account for the chance of fiber failure due to physical handling.

We have tested the agitator parameters to find if performance can be maintained at a minimized amplitude of oscillation, and for only one stage motion (two fiber bends instead of three), so as to maximize the lifetime of the agitator's mechanical assembly. Preliminary analysis shows that reducing the stage amplitude from $15 \mathrm{~cm}$ to $5 \mathrm{~cm}$, or $1 \mathrm{~cm}$ has little effect on modal-noise reduction, whereas driving two stages is about twice as effective as driving only one. In a similar test where we loosely wrapped six loops (of differing radii) of fiber around a 6 in diameter spool, and jiggled it by hand vertically $\sim 2 \mathrm{~cm}$, we find modal noise reduction comparable to that of the agitator. These tests indicate that the number of simultaneously varying bends in the fiber is more important than the amplitude of oscillation. While the Velmax units proved convenient for the prototype, we intend to replace the belt driven stages with reciprocating arms attached to motorized wheels which will result in small amplitudes and far gentler sinusoidal motions. To further minimize unnecessary wear-and-tear on the agitator and fibers we will pause agitation during science detector readouts. For example, the readout time for a 4-port, $4 \mathrm{kx} 4 \mathrm{k} \mathrm{CCD}$ at $100 \mathrm{kHz}$ is $\sim 40$ seconds. If there are 50 to 100 exposures taken a night, this approach would avoid 33 to 66 min of unnecessary fiber agitation every night. At the observatory, we will install the agitator within the "crypt", which is located below the telescope azimuth pivot, and connected to the basement hallway via the coude tunnel. This location was chosen both to minimize overall fiber cable length and to isolate the spectrometer from any vibrations caused by the moving agitator. Within the crypt, the agitator components will be directly attached to an existing $2 \mathrm{ft} \times 4 \mathrm{ft}$ optical table and be equipped with sensors that software can use to count cycles and verify that the agitator is operating correctly.

\subsection{Agitator Performance Testing}

Examples of laboratory tests with and without the KPF agitator are shown in Figures 4 and 5. Light from a HeNe laser at $543 \mathrm{~nm}$ is introduced to the optical train via a single mode optical fiber (see Figure 1). The distance along the fiber between the fiber input to the agitator is about $3 \mathrm{~m}$ and the distance from the agitator to the scrambler is about $19 \mathrm{~m}$. The fiber at the scrambler output is $6 \mathrm{~m}$ long. The upper panels show the static speckle pattern in the near-field where a loop in the fiber upstream of the agitator has is moved about $15 \mathrm{~cm}$ between images. Moving the fiber changes which modes of the fiber are propagated. The lower panels show again the near-field images, moving the fiber between images, but now with the agitator in motion. The smoothing of the speckle pattern is apparent in these images.

The image homogenization due to the agitator is further demonstrated by Figure 5. The images with, and without, the agitator are 2D Fourier transformed and radially averaged. In the near-field case, exposures are 7 sec, and the mid-to-high spatial frequencies are dramatically reduced by factors of a few hundred. The $\mathrm{X}$ axis is in spatial frequencies at the recording CCD; dividing by 9.3 gives spatial frequencies in the frame of the fiber tip. In the far-field case, even greater reduction of low-to-mid range spatial frequencies is observed. Note that higher spatial frequencies are quite low as the speckles in the far-field pattern are resolved by the camera. The $\mathrm{Y}$ axis is again in terms of the recording CCD camera; here, multiplying by $\sim 25$ gives spatial frequencies at the echelle grating pupil plane.

Within the KPF spectrometer, the fiber image is sliced into three slits by the reformatter, thus it is a matter of concern that speckle modes may shift from one slice into another where this could perturb the distribution of light (centroid) of the slices. Figure 6 shows the speckle image when the agitator is not operating and the three 

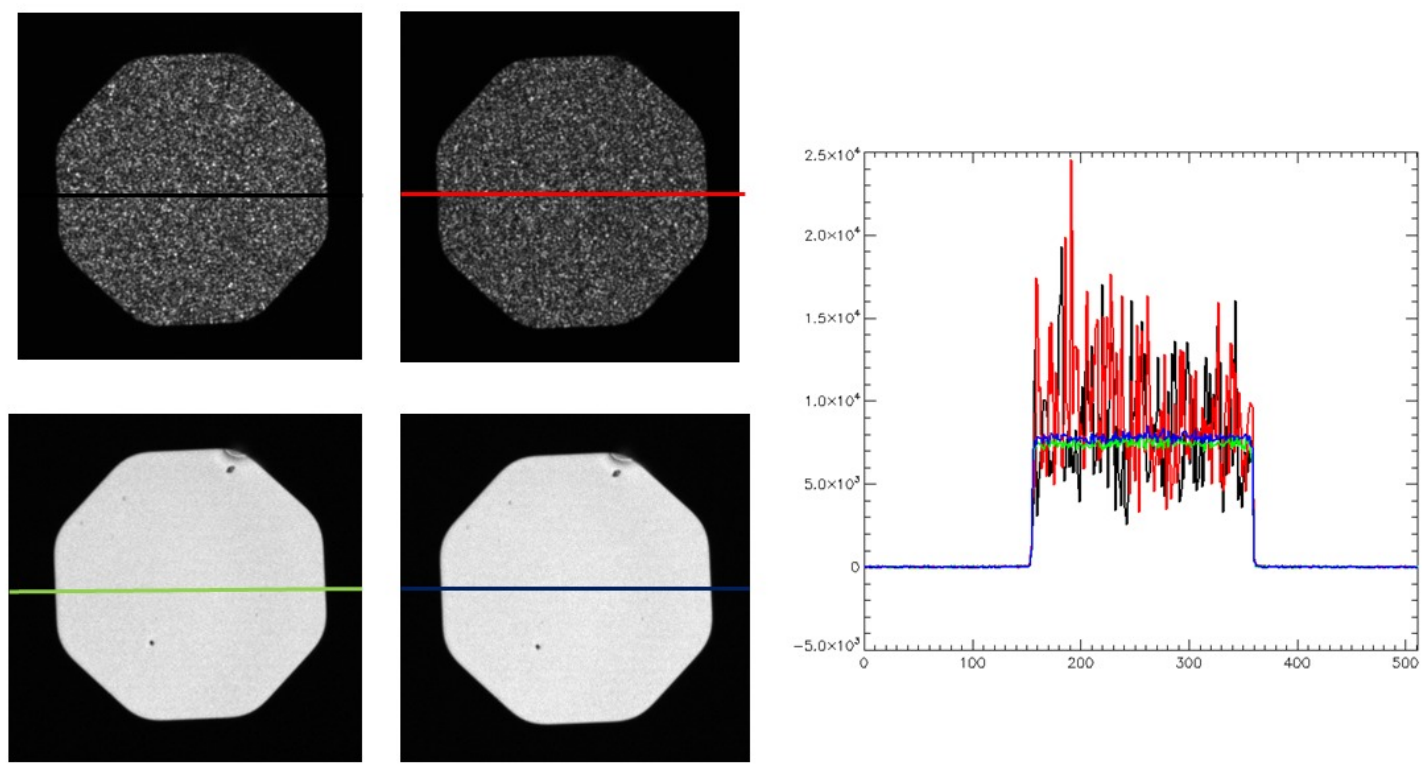

Figure 4. Images showing the effect of the agitator. The upper images show two near-field images without agitation where the fiber is moved between frames. The lower images show two successive images, with fiber movement, and with agitation. The graph shows single-row cross-sections from the center of each frame.
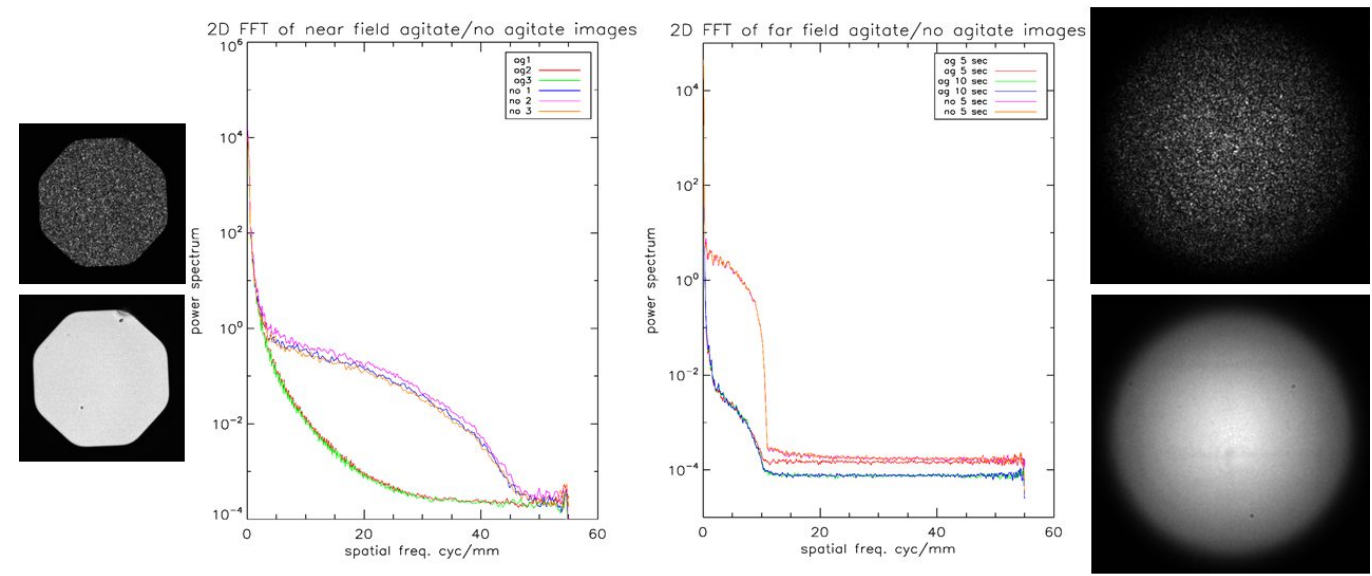

Figure 5. The effect of the agitator on image spatial frequency content. Near-field images with, and without, the agitator are shown on the left, and far-field images are shown on the right. The left graph shows the spatial power spectrum of near-field images and the reduction in mid-to-high spatial frequencies. The right graph shows the power spectrum of far-field images and the reduction of mid-to-low spatial frequencies. Higher spatial frequencies have low intensities as the speckles are resolved. The baseline shows some differences related to exposure time. 
slices used in the analysis. Figure 7 shows the image with agitation and the three slices. Here, the centroids of agitated fibers shift by less than $5 \mathrm{~nm}$ (as measured in the fiber frame of reference) during a 10 minute series of experiments. These analyses have not removed any drift of the fiber image due to drifts of the microscope lens and CCD positions. Note that the standard deviations of the slice centroids in the high resolution spectral direction are smaller by a factor of 3 than that of the unsliced fiber image. In addition, the standard deviation of this set of centroid measurements made with the agitator and a coherent laser source, is comparable to the long term stability measurements using the scrambler and LED source, described in Section 3.4.3. Therefore, the agitator is successfully mitigating image drifts caused by modal noise at the level of our measurement ability.

We verified the results outlined above by taking additional near-field data, first with the green LED (peak at $530 \mathrm{~nm}, \mathrm{FWHM}=35 \mathrm{~nm}$ ) without agitation, then with the HeNe laser at $543 \mathrm{~nm}$. The laser tests were performed with no agitation, hand agitation, and mechanical agitation at 17, 5, 1, and $0.5 \mathrm{~cm}$ amplitudes and provide a uniform data set with comparable counting statistics. Each test comprised 30 exposures of two second duration which allowed for the rapid acquisition of images to minimize any systematic errors caused by thermal drifts. The data are analyzed for both centroid stability and signal-to-noise (S/N), for the whole fiber and in three slices. We calculate $\mathrm{S} / \mathrm{N}$ in the manner of Petersburg et al. (Ref. 12). which is the median value of the image divided by the standard deviation of the difference of the image and median-filtered version of itself. Each image is first masked (by a few pixels fewer than the slices depicted in Figures 6 and 7) to exclude the edges where the count-rates change abruptly. Centroids are determined as the gravitational center-of-mass in each coordinate. We summarize these results in Table 1.

Table 1. Agitator Signal to Noise and Centroid Stability

\begin{tabular}{|c|c|c|c|c|c|c|c|}
\hline Test & $\begin{array}{l}\mathrm{S} / \mathrm{N}, \\
\text { Sliced }^{b}\end{array}$ & $\begin{array}{l}\text { X RMS } \\
(\mathrm{px})\end{array}$ & $\begin{array}{l}\text { Y RMS } \\
(\mathrm{px})\end{array}$ & $\begin{array}{l}\text { X Slice RMS } \\
1,2,3(\mathrm{px})\end{array}$ & $\begin{array}{l}\text { X Slice RMS } \\
\text { Scaled to } \\
\text { Slit Width }^{c}\end{array}$ & $\begin{array}{l}\text { Y Slice RMS } \\
1,2,3(\mathrm{px})\end{array}$ & N/Pixel \\
\hline Green LED & $\begin{array}{l}68.6 \\
67.8\end{array}$ & .0026 & .0060 & $\begin{array}{l}.0014 \\
.0020 \\
.0021\end{array}$ & $\begin{array}{l}.0042 \\
.0060 \\
.0063\end{array}$ & $\begin{array}{l}.0053 \\
.0093 \\
.0065\end{array}$ & 19,481 \\
\hline $\begin{array}{l}\text { No Agit } \\
\text { Laser }\end{array}$ & $\begin{array}{l}2.40 \\
2.41\end{array}$ & .36 & .29 & $\begin{array}{l}.20 \\
.20 \\
.19\end{array}$ & $\begin{array}{l}.0060 \\
.0060 \\
.0057\end{array}$ & $\begin{array}{l}.50 \\
.49 \\
.45\end{array}$ & 15,494 \\
\hline $\begin{array}{l}\text { Hand Agit } \\
\text { Laser }\end{array}$ & $\begin{array}{l}68.3 \\
67.5\end{array}$ & .0074 & .0061 & $\begin{array}{l}.0027 \\
.0049 \\
.0062\end{array}$ & $\begin{array}{l}.0081 \\
.0147 \\
.0186\end{array}$ & $\begin{array}{l}.0160 \\
.0064 \\
.0050\end{array}$ & 21,116 \\
\hline $\begin{array}{l}\text { Single Agit } \\
17 \mathrm{~cm} \\
\text { Laser }\end{array}$ & $\begin{array}{l}54.8 \\
52.8\end{array}$ & .0092 & .0094 & $\begin{array}{l}.0043 \\
.0062 \\
.0114\end{array}$ & $\begin{array}{l}.0129 \\
.0186 \\
.0342\end{array}$ & $\begin{array}{l}.0289 \\
.0112 \\
.0128\end{array}$ & 14,849 \\
\hline $\begin{array}{l}\text { Coupled Agit } \\
5 \mathrm{~cm} \\
\text { Laser }\end{array}$ & $\begin{array}{l}61.6 \\
61.0\end{array}$ & .0086 & .0079 & $\begin{array}{l}.0043 \\
.0047 \\
.0070\end{array}$ & $\begin{array}{l}.0129 \\
.0141 \\
.0210\end{array}$ & $\begin{array}{l}.0174 \\
.0069 \\
.0091\end{array}$ & 18,477 \\
\hline $\begin{array}{l}\text { Coupled Agit } \\
1 \mathrm{~cm} \\
\text { Laser }\end{array}$ & $\begin{array}{l}65.0 \\
64.5\end{array}$ & .0091 & .0069 & $\begin{array}{l}.0028 \\
.0050 \\
.0074\end{array}$ & $\begin{array}{l}.0084 \\
.0150 \\
.0222\end{array}$ & $\begin{array}{l}.0166 \\
.0101 \\
.0050\end{array}$ & 19,314 \\
\hline $\begin{array}{l}\text { Coupled Agit } \\
0.5 \mathrm{~cm} \\
\text { Laser }\end{array}$ & $\begin{array}{l}65.6 \\
65.1\end{array}$ & .0093 & .0086 & $\begin{array}{l}.0024 \\
.0047 \\
.0081\end{array}$ & $\begin{array}{l}.0072 \\
.0141 \\
.0243\end{array}$ & $\begin{array}{l}.0183 \\
.0086 \\
.0064\end{array}$ & 19,956 \\
\hline
\end{tabular}

${ }^{a}$ Ratio of fiber or slice width to measured X centroid RMS shift.

${ }^{b}$ First value is for the entire fiber, second value is the average of the 3 slices.

${ }^{c}$ Sliced centroid shift RMS translated to an unsliced instrument with the same resolution as KPF.

Contrary to expectations, we discovered that running the agitator at the smallest amplitudes produced the highest $\mathrm{S} / \mathrm{N}$ ratios! This is explained by the fact that at small amplitudes the agitator stages (driven at different 
frequencies) cycle through many direction changes per exposure causing the table it rests on to vibrate - this in turn wiggles the freely-hanging fibers where they enter and exit the agitator. In effect, we have created a coupled agitator that performs nearly as well as quasi-chaotic hand agitation. For the $17 \mathrm{~cm}$ amplitude case, we ran the agitator at a much slower speed, and placed foam blocks under the fibers where they enter and exit the agitator to prevent vibration. This mode behaves like a single agitator and shows a somewhat lower $\mathrm{S} / \mathrm{N}$.

In Figure 8 we present the $1 \mathrm{~cm}$ amplitude agitator-off $v s$ agitator-on centroids (left), and the agitator-on (right) for the unsliced case (black) and the sliced cases (colors). The average RMS (combining $1 \mathrm{~cm}$ and $0.5 \mathrm{~cm}$ data) of the unsliced X centroids is 0.0061 microns at the fiber, which corresponds to a KPF velocity error of 30 $\mathrm{cm} \mathrm{s}^{-1}$. The sliced X RMS centroids are about factor of $3 / \sqrt{3}$ smaller, the amount expected from a reduction factor of $1 / 3$ the slit width and $1 / 3$ the number of photons. This is a factor of $\sim 71$ smaller than the full-fiber agitator-off case and agrees with the previous results obtained with different data.
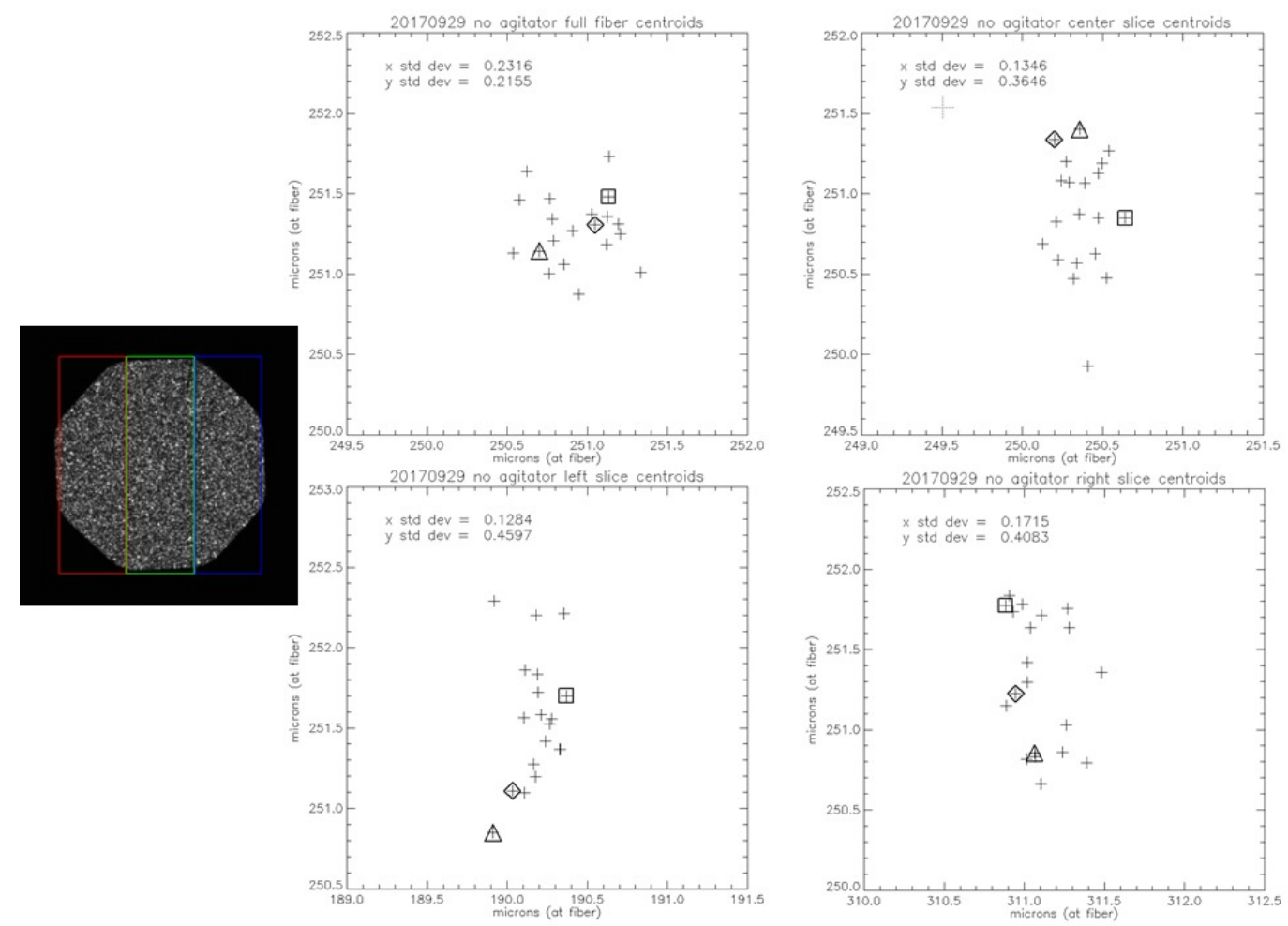

Figure 6. Centroids of the whole fiber image and the three slices without agitation. The image to the left shows the regions used in software to calculate the centroids. The plots show the centroids for a set of successive exposures. Moving clockwise from top left, the graphs show centroids of the whole image, central, right, and left slices. The $\mathrm{X}$ axis is the high spectral resolution direction. The diamond, triangle, and box symbols denote the results from the first, middle, and last images of the set. 

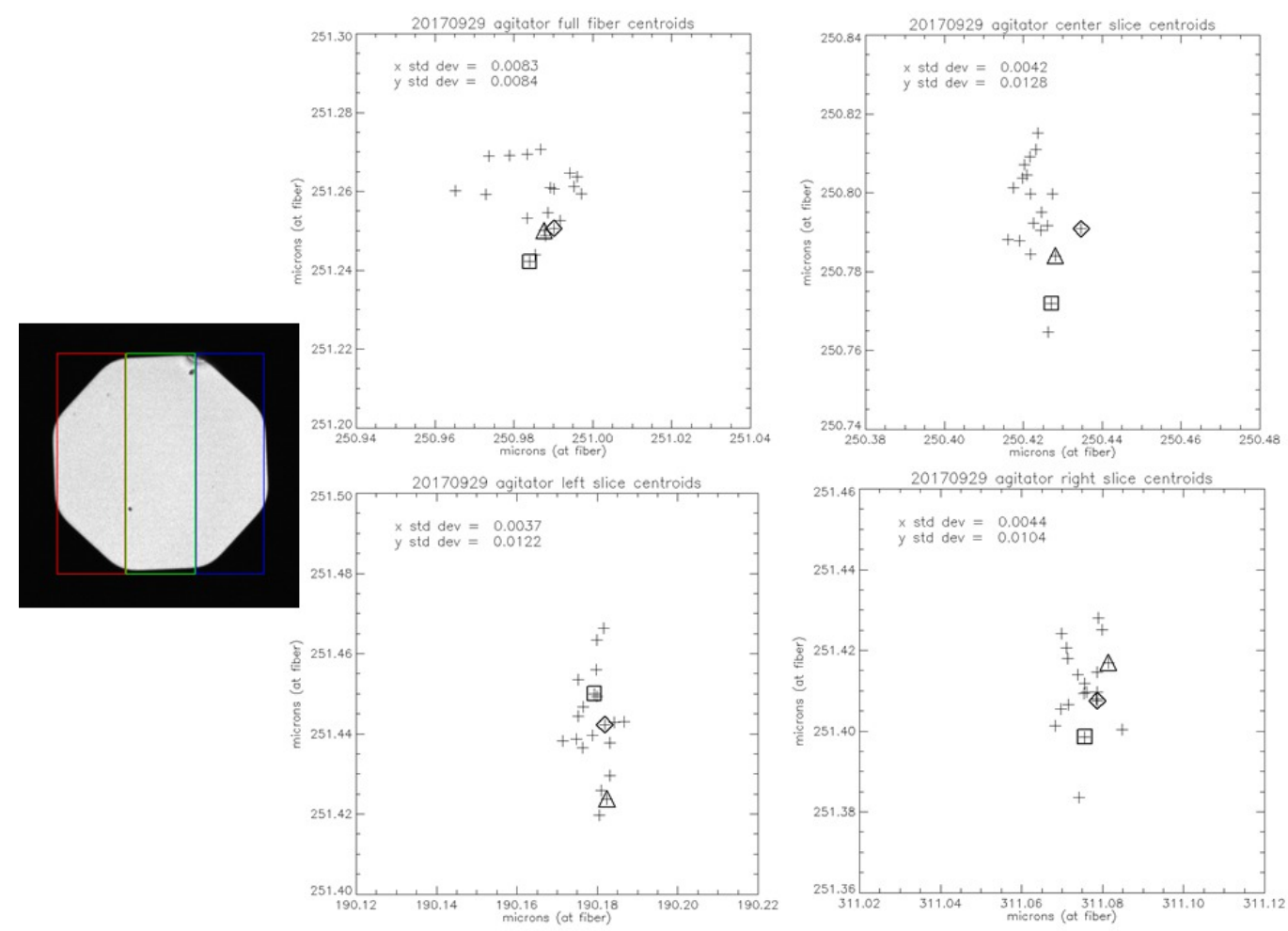

Figure 7. Centroids of the whole fiber image and the three slices with $1 \mathrm{~cm}$ agitation. The image to the left shows the regions used in software to calculate the centroids. The plots show the centroids for a set of successive exposures. The arrangement of the graphs, and the meaning of the symbols are as per Figure 6 . Note that the ranges plotted are $25 \mathrm{x}$ less than those without agitation shown in Fig. 6, and that slicing has reduced the RMS by an additional factor of $\sim 2$, or a total reduction in centroid drift of a factor $\sim 70$.
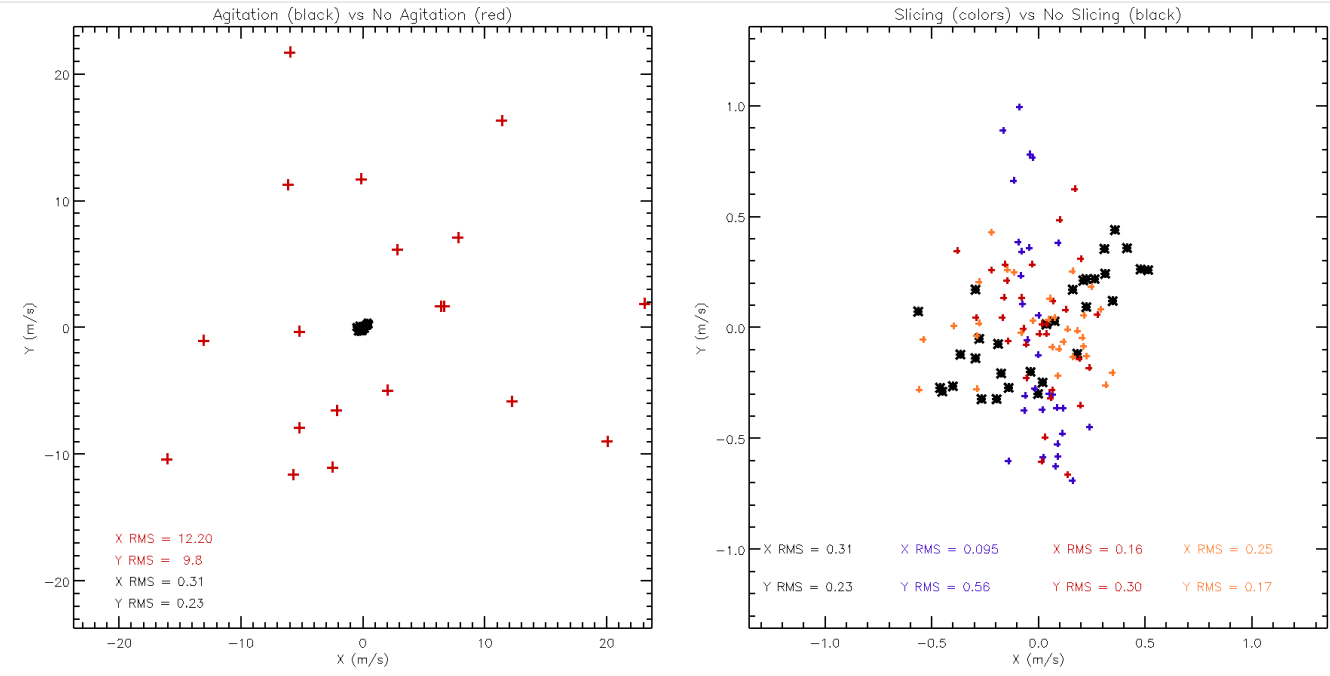

Figure 8. The left plot shows centroids for the whole fiber without agitation (red) with agitation (black) for the $1 \mathrm{~cm}$ amplitude data. The right plot shows the effect of slicing (colors) vs. no-slicing for the $1 \mathrm{~cm}$ agitated data. The $\mathrm{X}$ axis is the high spectral resolution direction. Compared to the unsliced centroid RMS, slicing has reduced the RMS in X by a factor of $3 / \sqrt{3}$. 


\section{SCRAMBLER}

\subsection{Design Choice}

We examined the twin-lens design and an alternative 'ball-lens' design. ${ }^{7}$ The twin-lens design was selected for a number of reasons. Firstly, the twin-lens system has lenses that are more easily anti-reflection coated than $\sim 1.5 \mathrm{~mm}$ diameter high index balls, and the twin-lens transmission is expected to be higher. Secondly, for the ball-lens system to perform properly, the input fiber must nearly touch the ball, with a tolerance of less than $10 \mu \mathrm{m}$, and this must be held stably. The tolerances of the twin lens system are more relaxed and departures from ideal positions can be compensated if needed. Thirdly, the fibers must pass into the spectrometer vacuum chamber and the twin lens system allows for transmission through a window. The ball-lens system requires a fiber vacuum feed-through which, due to inevitable contact with the fiber, will contribute to FRD. Finally, having the optical paths in air allows for a future simple switching system between fibers from Keck I and alternative fibers from Keck II.

\subsection{Optical Design}

Atmospheric turbulence causes variations in the stellar image at the fiber input, but these variations are dramatically reduced at the output due to scrambling. Octagonal cross-section fibers scramble light extremely well, but there are residual imprints of the intensity distribution at the fiber input that can be observed in the fiber output pattern. ${ }^{13,14}$ Optical fibers in general do a very good job of azimuthal far-field scrambling, but there persists a radial pattern. Octagonal fibers do an excellent job of scrambling the near-field illumination pattern, both azimuthally and radially; however, the far-field pattern retains a radial non-uniform pattern. The scrambler homogenizes both the near- and far-field intensity patterns.

A measure of the scrambling efficiency of a fiber system is the 'scrambling gain,'

$$
S G=\frac{\Delta d_{\text {input }} / D_{\text {input }}}{\Delta d_{\text {output }} / D_{\text {output }}}
$$

where, $\Delta d_{\text {input }}$ is the shift of the input spot on the fiber, $D_{\text {input }}$ is the fiber diameter, $\Delta d_{\text {output }}$ is the shift of the centroid of the output, and $D_{\text {output }}$ is the diameter of the fiber, or the pupil, image for near- and far-field measurements, respectively. The purpose of the "double-scrambler" is to swap the near-field and far-field patterns so that both the spectrometer slit and spectrometer pupil have very stable and uniform illumination, regardless of input star motions. ${ }^{15}$ Examples of near- and far- field images with and without the double scrambler are shown in Figure 13. Optical scramblers have been adopted by the latest generation of radial velocity spectrometers that use multi-mode fibers (e.g. References 7,16).

We present our "twin-lens" scrambler design in Figure 9. The optical system is analogous to that of a microscope with the first lens forming a magnified image of the fiber tip. The second lens acts as an eyepiece and it collimates the beam with a diameter equal to that of the fiber. In the present case, the lenses are identical Ross Optical L-AOC00 lenses with $12.5 \mathrm{~mm}$ focal lengths. The design shown is for the laboratory test system with $200 \mu \mathrm{m}$ fibers. The final KPF system with $225 \mu \mathrm{m}$ fibers uses the same lenses, but it will have a $\sim 10 \mathrm{~mm}$ smaller separation between the lenses, and $\sim 30 \mu \mathrm{m}$ larger fiber-to-lens distances. The KPF system utilizes a pair of twin-lens scramblers: one for the science and sky fibers and an additional system of similar design, with different components for the $100 \mu \mathrm{m}$ simultaneous calibration fiber of the spectrometer.

\subsection{Mechanical Design}

The scrambler system has been built and tested at SSL. It contains two parallel twin-lens systems and it consists of two robust aluminum flanges connected by a thick wall cylinder. One flange supports a vacuum window, and it will be mated to the spectrometer vacuum chamber. Each lens is axially secured by 3 ceramic balls on one face and 3 PEEK leaf springs on the opposite face. All contact occurs outside of the clear aperture. Each lens is secured radially by two machined ridges and one spring loaded Delrin ball. This style of lens retention is similar to mounts available from commercial devices such as those from Newport. Photographs of the scrambler assembly are shown in Figure 10. 

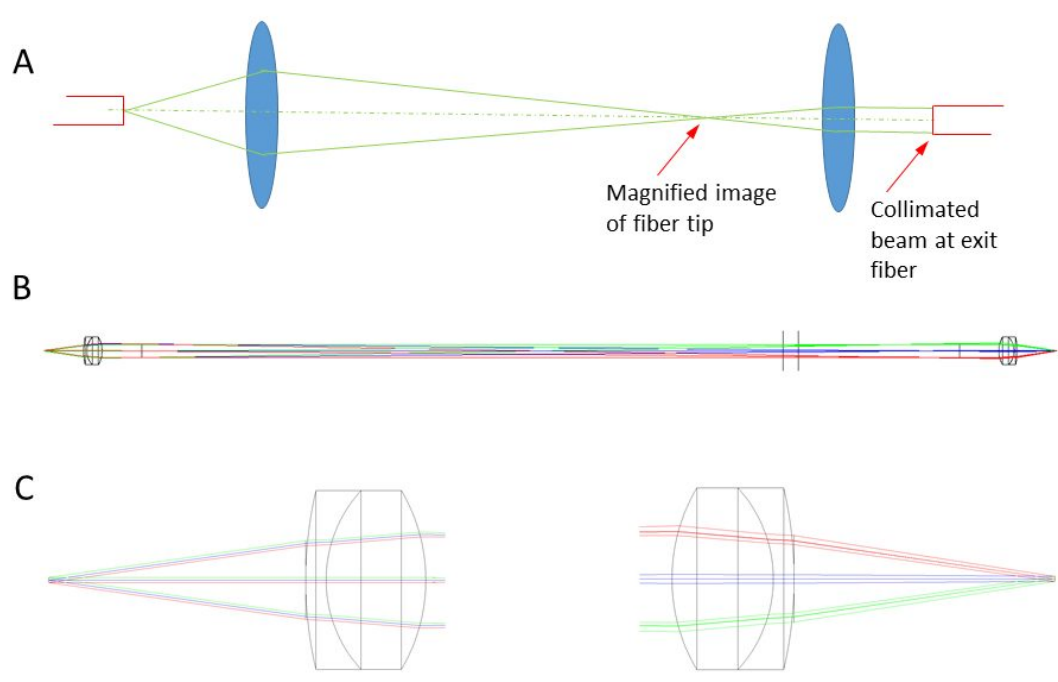

Figure 9. Optical diagram of twin-lens scrambler. A, a microscope analog of the scrambler. The first lens magnifies the fiber tip and the second lens collimates the light at the diameter of the fiber. B, the Zemax ray-trace of the system. A vacuum window is denoted by the vertical lines between the lenses. The overall length is $258 \mathrm{~mm}$. C, Enlarged views of the emitting tip at the left, and the converging collimated rays at the output at the right. The emitting $\mathrm{f} /$ cone is $\mathrm{f} / 3.5$ and the converging $\mathrm{f} /$ cone is $\sim \mathrm{f} / 3.4$.

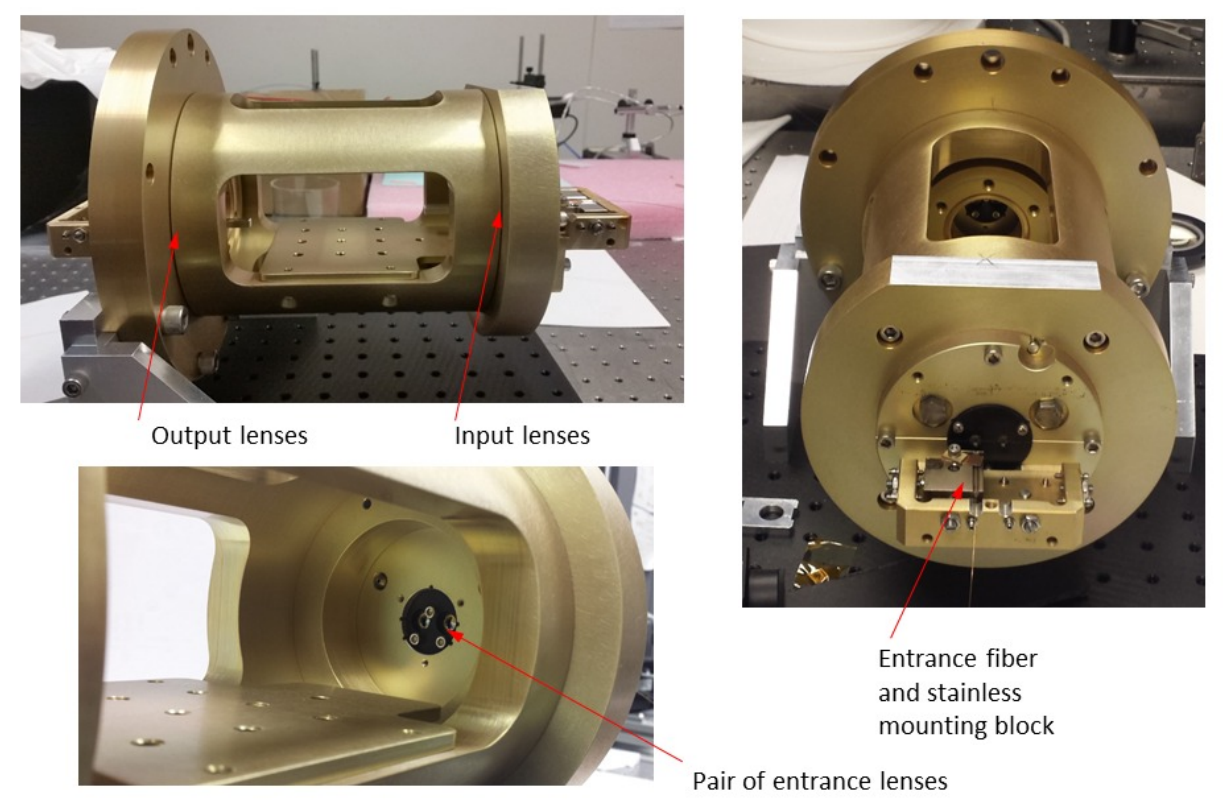

Figure 10. Photographs of the scrambler assembly. The upper left image shows a side view of the system with a small platform for adjustment optics. The lower left image shows the entrance lenses for the science and sky paths. The right image shows an oblique view along the axis. 
The fibers are mounted in glass ferrules which in turn are mounted in stainless steel blocks. These blocks are magnetically biased against six fine thread positioners $(0.2 \mathrm{~mm} / \mathrm{turn})$ that push against sapphire disks that are embedded and glued into the stainless blocks. This system provides fine adjustments and additionally allows the blocks to be easily dismounted for various fiber optic scrambling measurements. Figure 11 shows the scrambler during alignment.

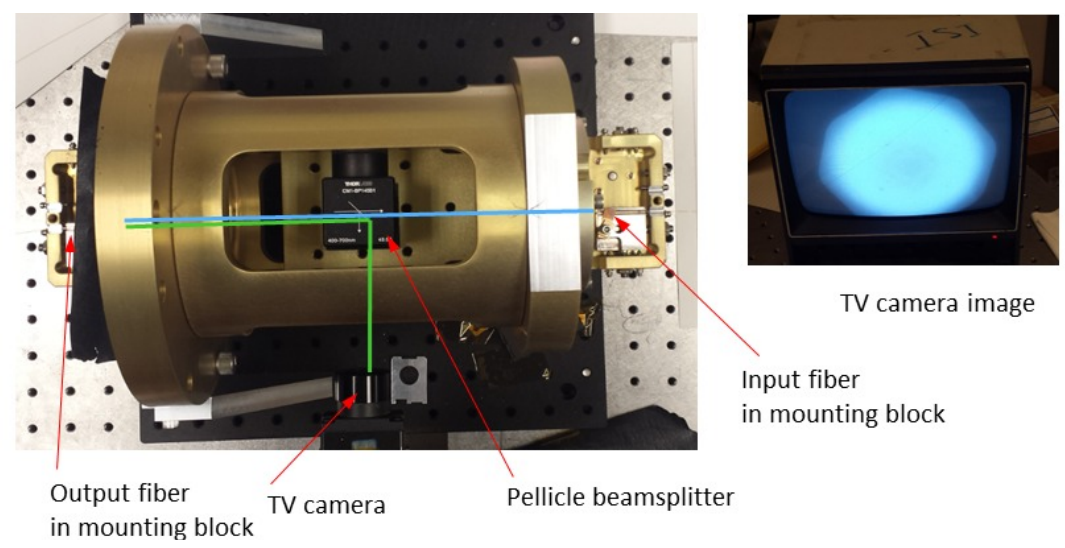

Figure 11. Photographs of the scrambler alignment. Light from the input fiber (blue) reflects off the output fiber face (green) and then the beamsplitter to a TV camera. The TV monitor shows both the output fiber octagon face and the 'doughnut' far-field pattern from the input fiber.

\subsection{Laboratory Scrambler Tests}

The design requirements of the scrambler are that it have a scrambling gain of $\geq 10,000$ and an exit f-cone from the output fiber of $>\mathrm{f} / 3.3$. The centroid of the near-field illumination pattern must be stable to $0.0225 \mu \mathrm{m}$ (referenced to the fiber frame), and the throughput stable to $0.25 \%$, over one hour time scales. We designed a variety of tests to determine the scrambler performance for each of these parameters. Most tests are performed first on single fibers, then in a fiber-scrambler-fiber configuration. Images are obtained with an ATIK 460 CCD monochrome camera with 2 by 2 pixel binning. For far-field images the fiber tip is typically placed $\sim 15 \mathrm{~mm}$ from the CCD. Near-field images are obtained by magnifying the fiber tip by a factor of 9.3 using a microscope objective. Since the CCD pixel size is $9.08 \mu \mathrm{m}$, the near-field image scale is very nearly $1 \mu \mathrm{m} /$ pixel. The individual scrambler tests are summarized in the following subsections.

\subsubsection{Fiber Illumination}

In order to analyze the scrambling properties of the fiber system in a realistic manner we illuminate our input fiber tip with light that simulates the geometry of the Keck telescope optics (see experimental setup in Figure 1). The light sources are broadband MIGHTEX LEDs (530 and $625 \mathrm{~nm}$ ), or a filtered narrow-band HgAr lamp $(543 \mathrm{~nm})$, that are injected into a $50 \mu \mathrm{m}$ core fiber, which then is the source for the test bed collimator. The collimated light is directed to an aperture mask scaled to simulate the Keck telescope mirror and secondary obscuration, followed by a f/3.7 lens which focuses the beam to match the output light cone of the KPF fiber injection unit (see Figure 12, left). Light is focused onto the input fiber tip creating a spot $50 \mu \mathrm{m}$ wide. The fiber is mounted on micrometer stages providing 5 degrees of freedom (X, Y, Z, Pitch, and Yaw).

\subsubsection{Scrambling Gain and Centroid Shifts caused by Fiber Translations and Tilts}

Scrambling gain, as defined by equation 2, relates the motion of the spot on the input fiber tip to the measured shifts in the images obtained at the fiber output. These shifts are measured for single fibers, and fiber-scramblerfiber combinations, and at near- and far-field. Figure 13 illustrates the illumination patterns for an unscrambled single fiber (top), and a scrambled two fiber system (bottom) at both far- and near-fields. 


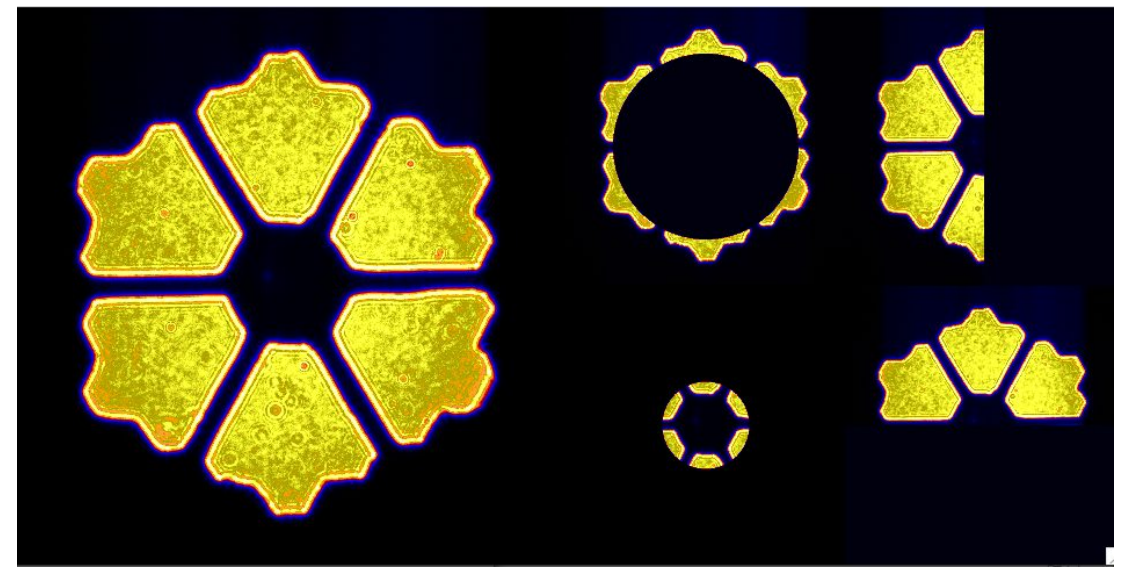

Figure 12. Image of the Keck full aperture (left), and several of the pupil masks employed for far-field FRD stability tests (right).
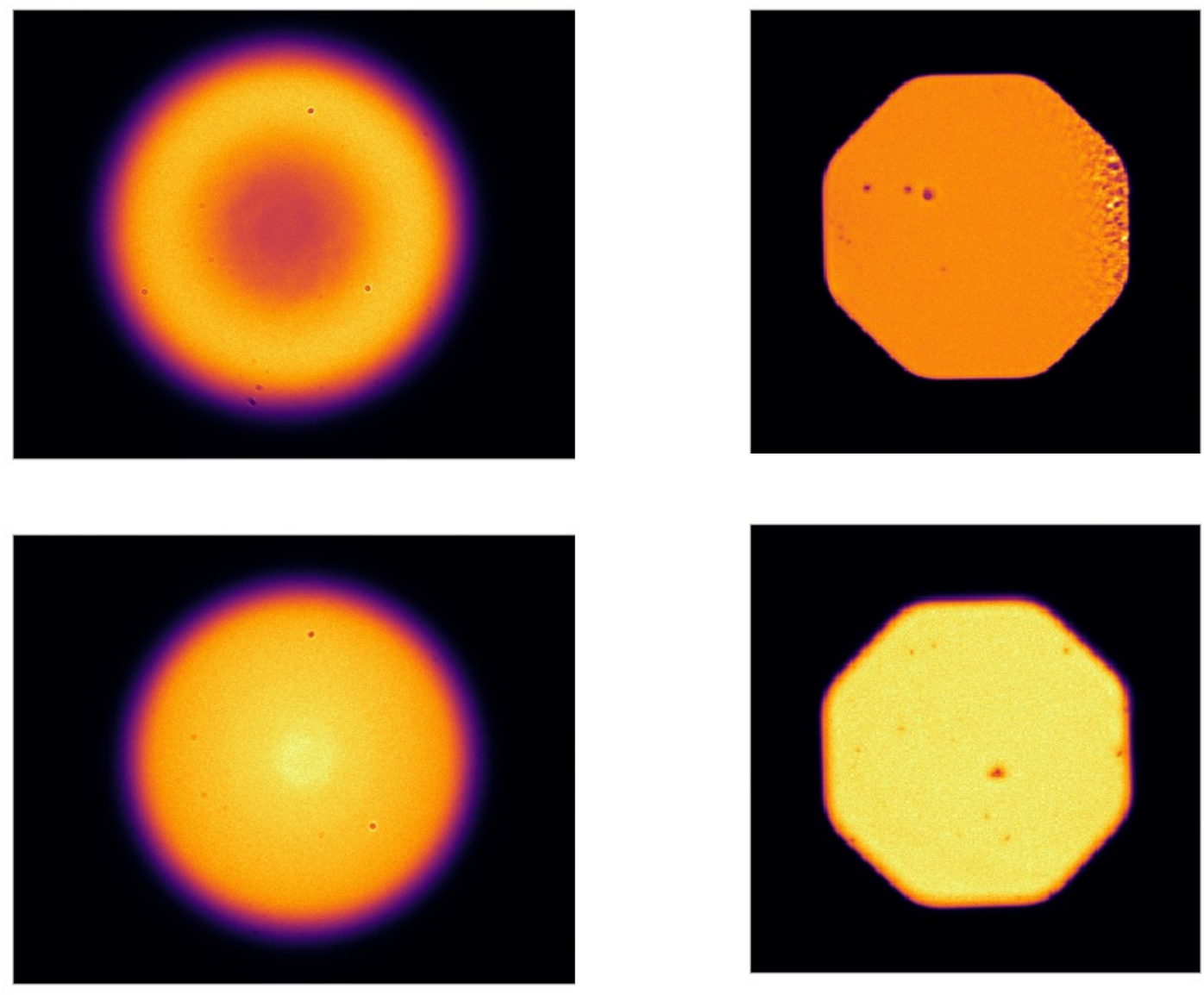

Figure 13. Examples of far- and near-field images (left, right) for a single $16 \mathrm{~m}$ fiber (top), and scrambled images (bottom) for a fiber-scrambler-fiber using $25 \mathrm{~m}$ input and $4 \mathrm{~m}$ output fibers. 
Translations of the input spot are done in $60 \mu \mathrm{m}$ steps in sets of three (one left, one center, and one right) and then repeated (typically two more times) Scrambling gains are then calculated within each set from the measured centroids and the known spot motions and fiber diameters. Figures 14 and 15 show far- and near-field centroidscatter plots for unscrambled (left), and scrambled data (right). The increase in scrambling gain is shown by the smaller scatter of the scrambled data as compared to single fiber data. The units in these graphs are $\mu \mathrm{m}$ as measured from centroids of images recorded by the CCD. Additional tests were performed by translating the input spot in steps of 70,80 , and $90 \mu \mathrm{m}$. The near-field average scrambling gain of all translation data is 19,400 \pm 3200 , and the average centroid shift $0.0087 \mu \mathrm{min}$ the output fiber frame. We note that this test is an extreme case, as in practice the stellar image at the input of our science fiber will be much larger than $50 \mu \mathrm{m}$, and the tip/tilt mirror in the KPF fiber injection unit will maintain the stellar image to within $10 \mu \mathrm{m}$ of the fiber center.
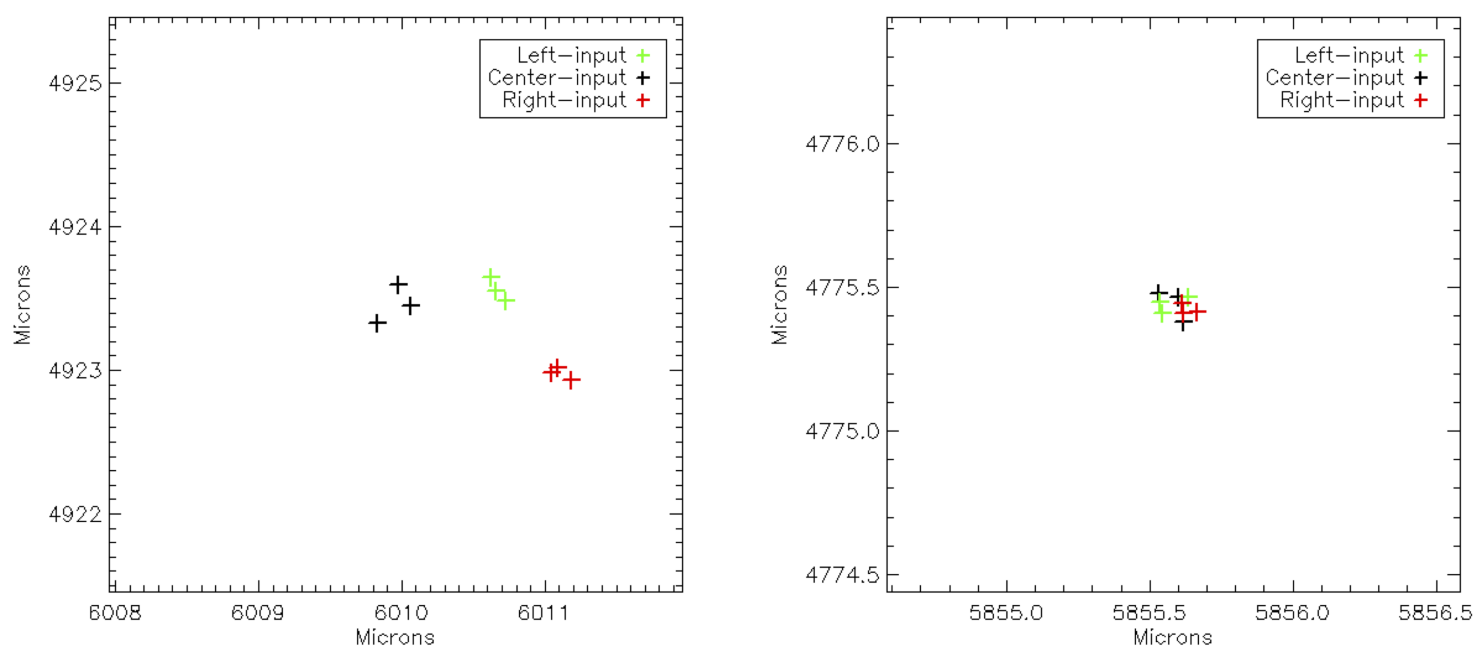

Figure 14. Far-field centroids determined by translating in $\mathrm{Z} \pm 60 \mu \mathrm{m}$ away from nominal of an unscrambled $25 \mathrm{~m}$ fiber with an average shift of $0.901 \mu \mathrm{m}$ and a scrambling gain of 3955 (left) 2017-08-03, and scrambled fibers with $25 \mathrm{~m}$ input and $6 \mathrm{~m}$ output with an average shift of $0.077 \mu \mathrm{m}$ and a scrambling gain of 51,300 (right) 2017-08-03.
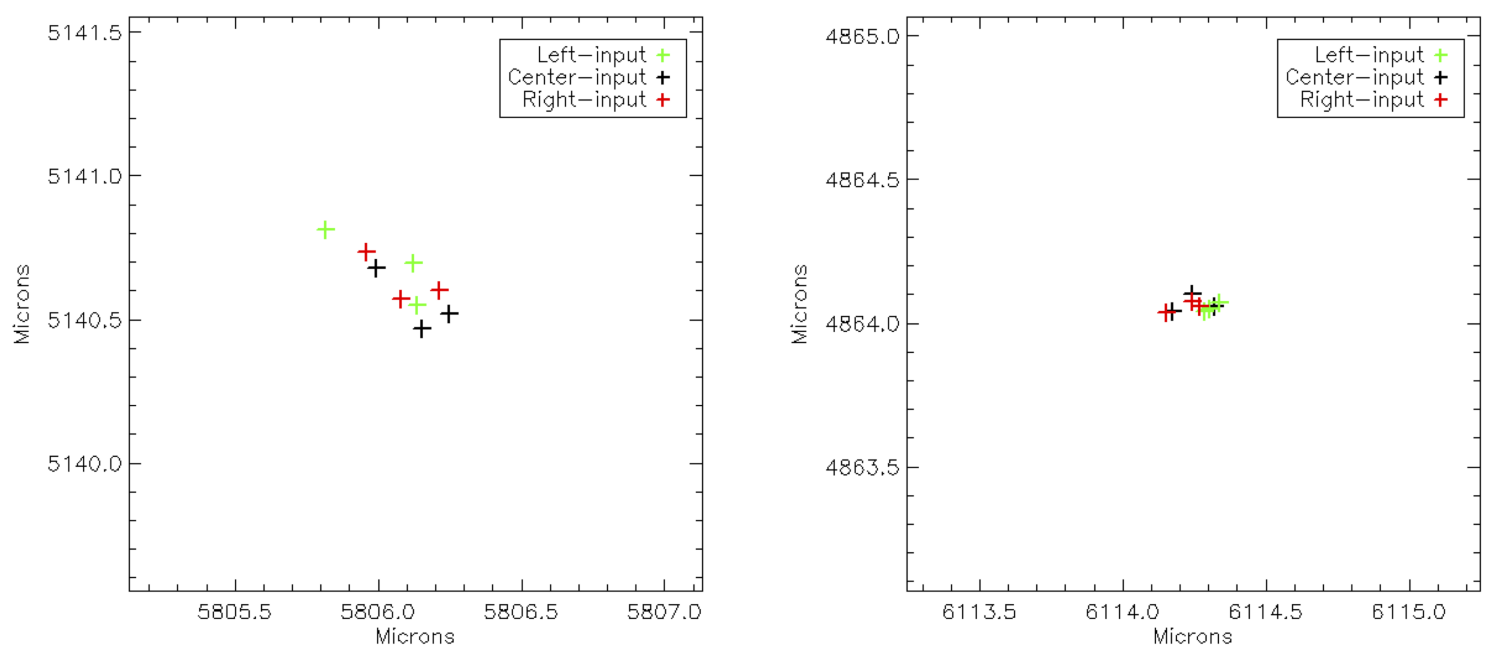

Figure 15. Near-field centroids determined by translating in $\mathrm{Z} \pm 60 \mu \mathrm{m}$ away from nominal of unscrambled $25 \mathrm{~m}$ fiber with an average shift of $0.129 \mu \mathrm{m}$ and a scrambling gain of 7236 (left) 2017-08-02, and scrambled fibers with $25 \mathrm{~m}$ input and $6 \mathrm{~m}$ output with an average shift of $0.083 \mu \mathrm{m}$ and a scrambling gain of 13,441 (right) 2017-08-01. 
Tilt tests are performed in an analogous manner as translation tests except that the fiber normal is tilted away from the optic axis by a known amount (0.2, 0.4 , and 0.6 degrees). We show the most extreme case of 0.6 degrees in Figures 16 and 17 for far- and near-field, unscrambled (left) and scrambled (right) cases. The smaller angle cases also showed comparable scrambling indicating that injecting light into our fibers at angles up to 0.6 degrees from normal does not compromise scrambling performance.
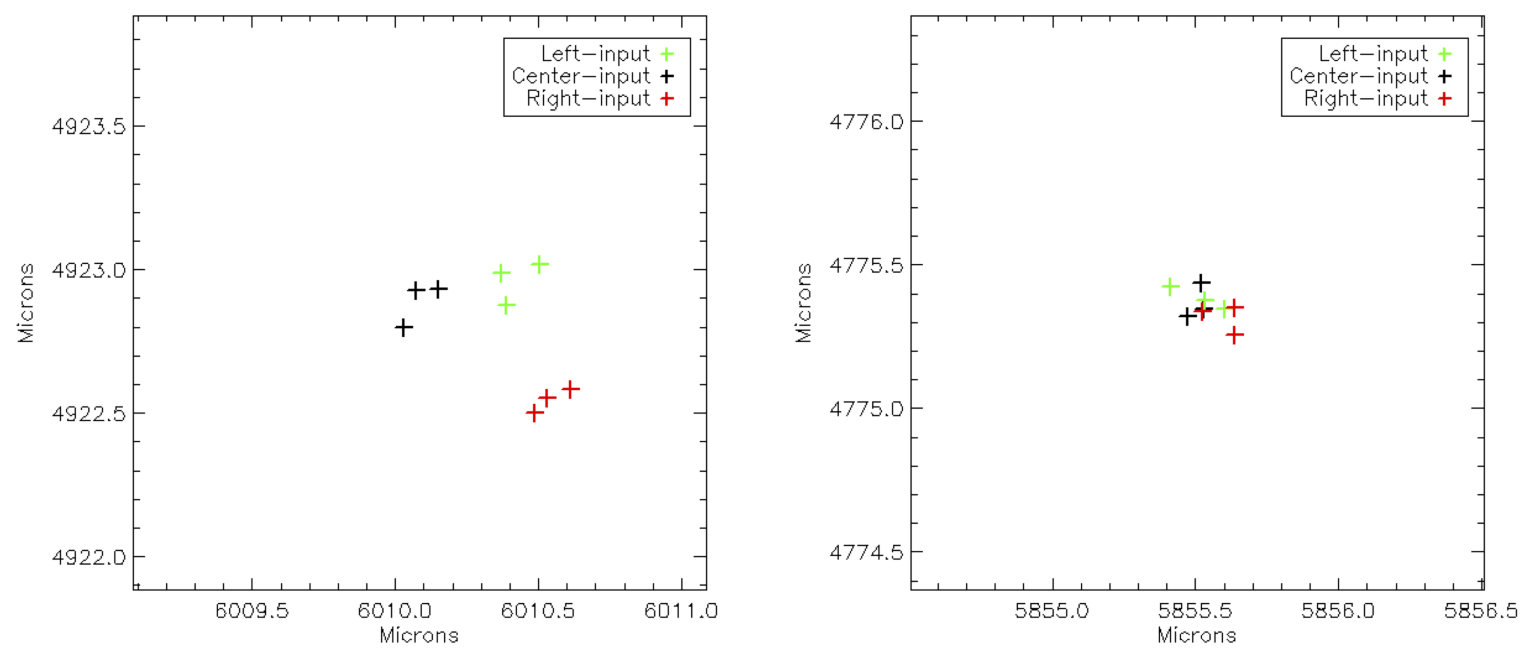

Figure 16. Far-field centroids due to tilting the injection angle \pm 0.6 degrees from fiber normal. The left graph, from a single $25 \mathrm{~m}$ fiber, has an average centroid shift of $0.45 \mu \mathrm{m}$. The right graph shows results from the $25 \mathrm{~m}$-scrambler- $6 \mathrm{~m}$ fiber system with an average centroid shift of $0.115 \mu \mathrm{m}$.
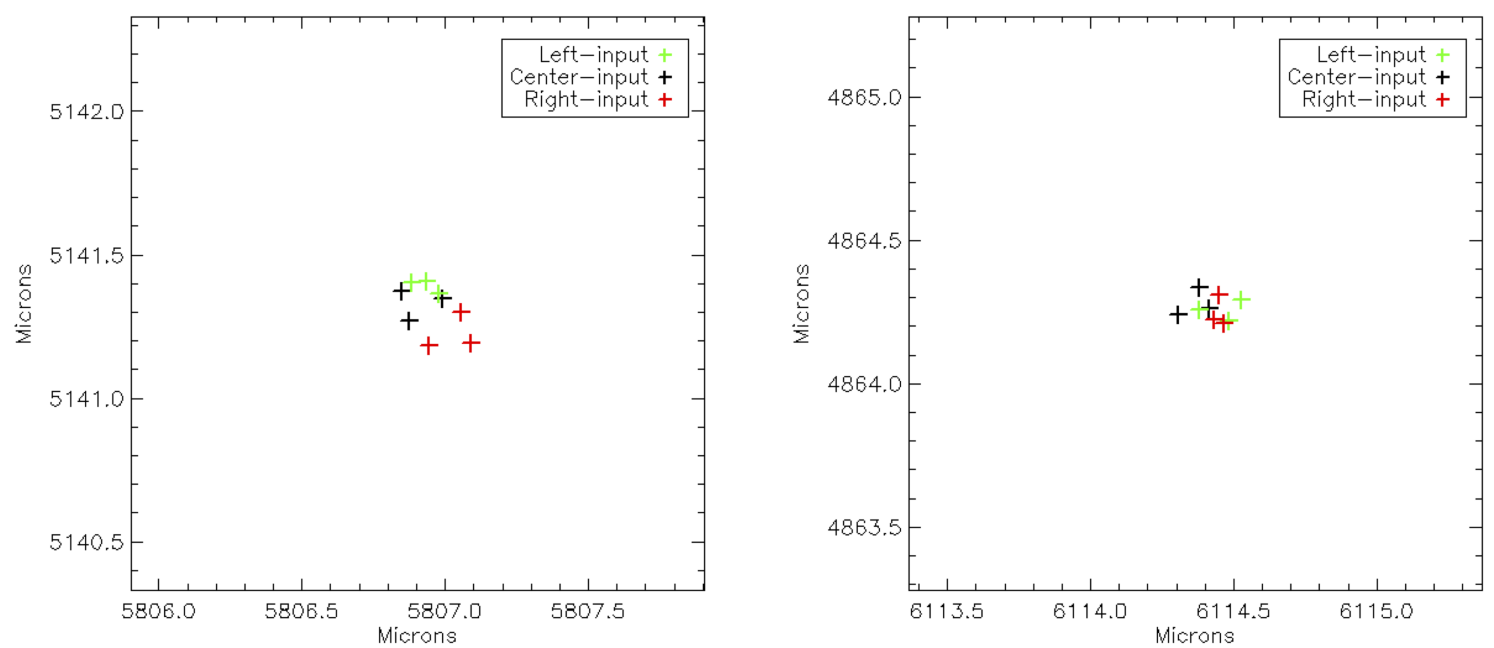

Figure 17. Near-field centroids due to tilting the injection angle \pm 0.6 degrees from fiber normal. The left graph, from a single $25 \mathrm{~m}$ fiber, shows an average centroid shift of $0.16 \mu \mathrm{m}$. The right graph shows results from the $25 \mathrm{~m}$-scrambler- $6 \mathrm{~m}$ fiber system with an average centroid shift of $0.104 \mu \mathrm{m}$. 


\subsubsection{Scrambler and Throughput Stability}

We performed many long-term scrambler stability tests in both far- and near-field. In the near-field case these tests showed centroid drifts of up to a few tenths of a micron at the fiber face. However, by utilizing an edgefinding algorithm (which is sensitive only to the edges of the near-field octagonal images, not brightness variations within), we were able to decouple drifts from our camera-microscope system from those caused by scrambler instability. Figure 18 shows near-field data obtained over a 22.5 hour period at 15 minute intervals. Centroids are determined with the edge-finder and with a moment-weighted mean (gravitational center-of-mass) techniques. The units are microns as measured at the fiber tip. The RMS drift in $\mathrm{X}$ is $0.11 \mu \mathrm{m}$ and represents the bulk motion of the octagonal images. The difference between the two centroid sets represents the illumination drift caused by scrambler instability. The RMS is $0.007 \mu \mathrm{m}$ which is only slightly larger than our measuring limit of $0.005 \mu \mathrm{m}$.
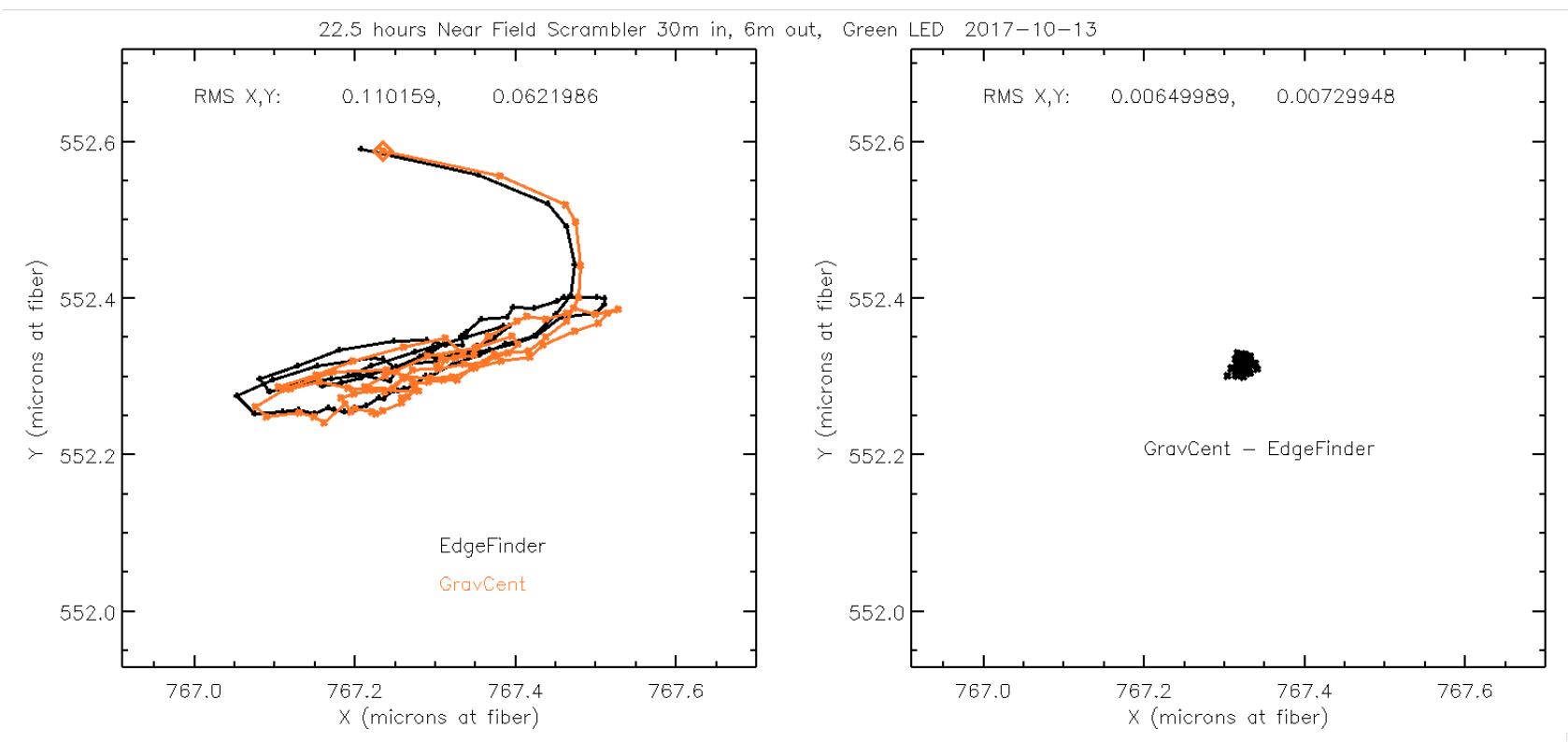

Figure 18. Long-term stability test centroids (left) using edge-finder (black) and gravitational-center (red) algorithms. Differences between the gravitational and edge-finder centroids (right) indicate scrambler stability of $\sim 0.007 \mu \mathrm{m}$.

Temperatures of the optical bench were also obtained during the long-term test. In Figure 19 we show the measured gravitational centroids and temperature vs. time (temperature was not controlled during the test and followed laboratory temperature). The positional drifts are clearly correlated with temperature changes of \pm 1 $\mathrm{C}$ and, for near field data, are removed using the edge-finding technique. Therefore, the largest observed drifts were caused by the test setup motions of the output fiber with respect to the CCD. For far-field data, drifts caused by temperature variations are mitigated by only comparing images taken in short time intervals (typically under 2 minutes). The fourth panel plots the corresponding total counts for each image. The RMS variation is less than $0.1 \%$ indicating high throughput stability.

In addition to far-field centroid stability, we also require stable FRD (f-cone) of the output fiber. We verify this by placing various aperture masks at the secondary obstruction that only allow extreme angle rays, or only rays from the left, right, top, or bottom of the aperture (see right panels of Figure 12). The measured RMS of the f-cone of full, left, right, top, and bottom apertures is $0.015 \%$, indicating minimal change in FRD. The change in $\mathrm{f}$-cone between the extreme angle apertures is $1.4 \%$.

\subsection{Absolute Scrambler Throughput}

By comparing the total counts emanating from the scrambler input fiber in a given exposure time to those from the scrambler output fiber, an absolute efficiency of $86.3 \%$ is obtained for the scrambler-plus-output fiber configuration. The scrambler in this laboratory setup utilizes two $\mathrm{MgF}_{2}$ coated lenses and an un-coated output 

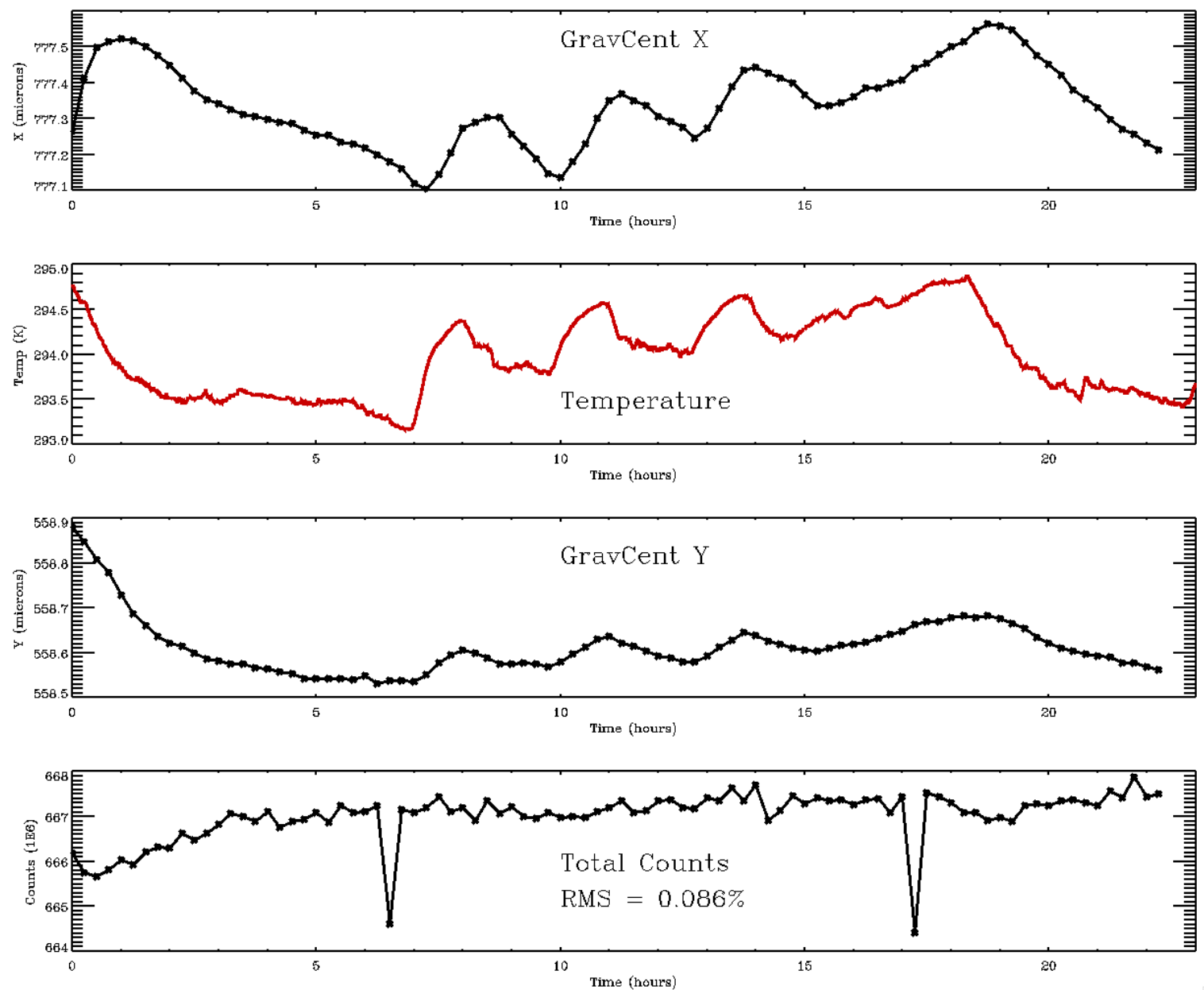

Figure 19. Gravitational centroids for long-term stability test in X and Y (first and third panels) plotted with temperature (red, second panel). Fourth panel shows throughput stability of $<0.1 \%$ over the 22.5 hour period.

fiber $6 \mathrm{~m}$ long. The vacuum window was not installed. When taking into account reflection and length-losses, the expected scrambler efficiency is $86.9 \%$. Thus, the scrambler is performing at $99.3 \%$ of its expected throughput value demonstrating correct alignment and minimal FRD losses. For the final design utilizing Ross Optical 220 AR coatings on the two scrambler lenses and vacuum window, and both ends of the output fiber, we expect an absolute scrambler-plus- $6 \mathrm{~m}$ output fiber efficiency of $\sim 92 \%$ at $530 \mathrm{~nm}$.

\subsection{Scrambler Performance: Summary and Future Work}

The tests performed so far show that the scrambler is meeting all its design requirements. We summarize scrambler performance in Table 2. However, additional tests are required to ensure a complete understanding of the agitator-scrambler system. These studies include:

- Scrambler optical FRD: Test and optimize the FRD performance dependence on the distance between the scrambler lenses.

- Examine the long term stability of far-field images: Studies have been performed of the long term stability of the scrambler with near-field images, but far-field studies must also be performed. In addition, the performance will be measured for fiber movements similar to those experienced during telescope observations. 
- Vacuum performance of the scrambler: Mount the vacuum window to the scrambler flange and mount this flange to a vacuum chamber to check that the system remains aligned under vacuum. Zemax evaluation indicates that the vacuum window will not degrade performance.

- Fiber performance in protective cables: Studies will be performed to validate the FRD performance of fibers in cables.

- Long term agitator tests: Ensure that the agitator system performs reliably and that fibers under repetitive bend stresses do not degrade FRD performance (note that 500,000 cycle fiber stress tests have been successfully performed by the Dark Energy Spectroscopic Instrument ${ }^{17}$ ).

Table 2. Scrambler Parameters

\begin{tabular}{ccc}
\hline Parameter & Value & Requirement \\
\hline Near-Field Scrambling Gain $^{a}$ & $19,400 \pm 3200$ & $\geq 10,000$ \\
Centroid Shifts at Fiber $^{b}$ & $<0.01 \mu \mathrm{m}$ & \\
Far-Field Scrambling Gain $^{b}$ & $>40,000$ & \\
Observed Shifts at 200 mm Pupil & & \\
Output f-cone & $2.5 \mu \mathrm{m}$ & \\
Centroid Stability $/$ wrt Fiber Position $^{c}$ & $0.007 \mu \mathrm{m}$ & $\geq \mathrm{f} / 3.3$ \\
Throughput Stability $^{c}$ & $<0.1 \%$ & \\
Throughput $^{d}$ & $86.3 \%$ & $>80 \%$ \\
\hline
\end{tabular}

${ }^{a}$ Average for input fiber spot translations of $\pm 60,70,80$, and $90 \mu \mathrm{m}$.

${ }^{b}$ Average value of observed output fiber centroid shifts from translation, tilt, and fiber motion tests.

${ }^{c}$ Over 22 hours and temperature range of $\pm 1 \mathrm{C}$.

${ }^{d}$ Through scrambler and one uncoated $6 \mathrm{~m}$ fiber at $530 \mathrm{~nm}$.

\section{CONCLUSIONS}

We have designed and tested a system to spatially and temporally homogenize the illumination of the KPF spectrograph entrance aperture. Scrambling gains of $>16,000$ in the near-field and $>40,000$ in the far-field are achieved. Modal-noise power is reduced by a factor of $\sim 300$ in the near-field, $\sim 1000$ in the far-field. Agitator near-field data show Signal-to-Noise ratios that approach values obtained from hand agitaion and from a green LED in 2 second exposures. Near-field image centroids are stable to .01 $\mu \mathrm{m}$ at the fiber tip. for both input fiber translation tests and input fiber tilt tests. Overnight far-field tests show f-cone stability to $0.015 \%$. Half-sector pupil mask tests also show f-cone stability of $0.015 \%$. Absolute scrambler throughput is within $0.7 \%$ of predicted maximum indicating correct alignment and no light-loss from FRD. Long-term scrambler tests show throughput variations of $<0.1 \%$.

When coupled to the KPF spectrograph the scrambler and agitator are showing a velocity stability of 0.30 $\mathrm{m} \mathrm{s}^{-1}$. Slicing reduces near-field centroid RMS by a factor of $3 / \sqrt{3}$. This increase in velocity stability may be realized in the final instrument if there is no cross-talk between the three slices.

\section{ACKNOWLEDGMENTS}

The authors thank the Heising-Simons Foundation, the W.M. Keck Foundation, the University of California, Berkeley, the California Institute of Technology, and the University of Hawaii for financial support of KPF. We also thank Claire Poppett and Sharon Jelinsky of the DESI project for help bonding ferrules onto our fibers. This work was performed by SPH under contract with the Jet Propulsion Laboratory (JPL) funded by NASA through the Sagan Fellowship Program executed by the NASA Exoplanet Science Institute. 


\section{REFERENCES}

[1] Pepe, F. A. and Lovis, C., "From HARPS to CODEX: exploring the limits of Doppler measurements," Physica Scripta Volume T 130, 014007 (Aug. 2008).

[2] Heacox, W. D., "On the application of optical-fiber image scramblers to astronomical spectroscopy," AJ 92, 219-229 (July 1986).

[3] Roy, A., Halverson, S., Mahadevan, S., and Ramsey, L. W., "Scrambling and modal noise mitigation in the Habitable Zone Planet Finder fiber feed," in [Ground-based and Airborne Instrumentation for Astronomy V], Proc. of the SPIE 9147, 91476B (July 2014).

[4] Gibson, S. R., Howard, A. W., Roy, A., Smith, C., Halverson, S., Edelstein, J., Kassis, M., Wishnow, E. H., Raffanti, M., Allen, S., Chin, J., Coutts, D., Cowley, D., Curtis, J., Deich, W., Finstad, D., Feger, T., Gurevich, Y., Ishikawa, Y., James, E., Jhoti, E., Lanclos, K., Lilley, S., Miller, T., Milner, S., Payne, T., Rider, K., Rockosi, C., Sanford, D., Schwab, C., Seifahrt, A., Sirk, M. M., Smith, R., Stuermer, J., Weisfeiler, M., Wilcox, M., and Vandenberg, A., "Keck Planet Finder: Preliminary Design," in [Groundbased and Airborne Instrumentation for Astronomy VI], Proc. of the SPIE 10702 (Aug. 2018).

[5] Mahadevan, S., Halverson, S., Ramsey, L., and Venditti, N., "Suppression of Fiber Modal Noise Induced Radial Velocity Errors for Bright Emission-line Calibration Sources," ApJ 786, 18 (May 2014).

[6] Baudrand, J. and Walker, G. A. H., "Modal Noise in High-Resolution, Fiber-fed Spectra: A Study and Simple Cure," PASP 113, 851-858 (July 2001).

[7] Halverson, S., Roy, A., Mahadevan, S., Ramsey, L., Levi, E., Schwab, C., Hearty, F., and MacDonald, N., "An Efficient, Compact, and Versatile Fiber Double Scrambler for High Precision Radial Velocity Instruments," ApJ 806, 61 (June 2015).

[8] Halverson, S., Mahadevan, S., Ramsey, L., Terrien, R., Roy, A., Schwab, C., Bender, C., Hearty, F., Levi, E., Osterman, S., Ycas, G., and Diddams, S., "The habitable-zone planet finder calibration system," in [Ground-based and Airborne Instrumentation for Astronomy V], Proc. of the SPIE 9147, 91477Z (Aug. 2014).

[9] Plavchan, P. P., Bottom, M., Gao, P., Wallace, J. K., Mennesson, B., Ciardi, D., Crawford, S., Lin, S., Beichman, C., Brinkworth, C., Johnson, J., Davison, C., White, R., Anglada-Escude, G., von Braun, K., Vasisht, G., Prato, L., Kane, S., Tanner, A., Walp, B., and Mills, S., "Precision near-infrared radial velocity instrumentation II: noncircular core fiber scrambler," in [Techniques and Instrumentation for Detection of Exoplanets VI], Proc. of the SPIE 8864, 88640G (Sept. 2013).

[10] McCoy, K. S., Ramsey, L., Mahadevan, S., Halverson, S., and Redman, S. L., "Optical fiber modal noise in the 0.8 to 1.5 micron region and implications for near infrared precision radial velocity measurements," in [Ground-based and Airborne Instrumentation for Astronomy IV], Proc. of the SPIE 8446, 84468J (Sept. 2012).

[11] Glaesemann, G. S., Dainese, P., Merrion, E., and Jabulani, D., "The Mechanical Reliability of Corning Optical Fiber in Small Bend Scenarios," tech. rep., Corning Inc. (Dec. 2007).

[12] Petersburg, R. R., McCracken, T. M., Eggerman, D., Jurgenson, C. A., Sawyer, D., Szymkowiak, A. E., and Fischer, D. A., "Modal Noise Mitigation through Fiber Agitation for Fiber-fed Radial Velocity Spectrographs," ApJ 853, 181 (Feb. 2018).

[13] Avila, G., "FRD and scrambling properties of recent non-circular fibres," in [Ground-based and Airborne Instrumentation for Astronomy IV], Proc. of the SPIE 8446, 84469L (Sept. 2012).

[14] Spronck, J. F. P., Kaplan, Z. A., Fischer, D. A., Schwab, C., and Szymkowiak, A. E., "Extreme Doppler precision with octagonal fiber scramblers," in [Ground-based and Airborne Instrumentation for Astronomy IV], Proc. of the SPIE 8446, 84468T (Sept. 2012).

[15] Hunter, T. R. and Ramsey, L. W., "Scrambling properties of optical fibers and the performance of a double scrambler," PASP 104, 1244-1251 (Dec. 1992).

[16] Avila, G., Kohler, D., Araya, E., Gilliotte, A., and Eckert, W., "Performances of HARPS and FEROS fibers in La Silla ESO Observatory," in [Ground-based Instrumentation for Astronomy], Moorwood, A. F. M. and Iye, M., eds., Proc. of the SPIE 5492, 669-676 (Sept. 2004).

[17] DESI Collaboration, Aghamousa, A., Aguilar, J., Ahlen, S., Alam, S., Allen, L. E., Allende Prieto, C., Annis, J., Bailey, S., Balland, C., and et al., "The DESI Experiment Part II: Instrument Design," ArXiv e-prints (Oct. 2016). 